\title{
Cluster Variables on Certain Double Bruhat Cells of Type $(u, e)$ and Monomial Realizations of Crystal Bases of Type $A^{\star}$
}

\author{
Yuki KANAKUBO and Toshiki NAKASHIMA \\ Division of Mathematics, Sophia University, Yonban-cho 4, Chiyoda-ku, \\ Tokyo 102-0081, Japan \\ E-mail: j_chi_sen_you_ky@sophia.ac.jp,toshiki@sophia.ac.jp
}

Received October 01, 2014, in final form April 14, 2015; Published online April 23, 2015

http://dx.doi.org/10.3842/SIGMA.2015.033

\begin{abstract}
Let $G$ be a simply connected simple algebraic group over $\mathbb{C}, B$ and $B_{-}$be two opposite Borel subgroups in $G$ and $W$ be the Weyl group. For $u, v \in W$, it is known that the coordinate ring $\mathbb{C}\left[G^{u, v}\right]$ of the double Bruhat cell $G^{u, v}=B u B \cap B_{-} v B_{-}$is isomorphic to an upper cluster algebra $\overline{\mathcal{A}}(\mathbf{i})_{\mathbb{C}}$ and the generalized minors $\{\Delta(k ; \mathbf{i})\}$ are the cluster variables belonging to a given initial seed in $\mathbb{C}\left[G^{u, v}\right]$ [Berenstein A., Fomin S., Zelevinsky A., Duke Math. J. 126 (2005), 1-52]. In the case $G=\mathrm{SL}_{r+1}(\mathbb{C}), v=e$ and some special $u \in W$, we shall describe the generalized minors $\{\Delta(k ; \mathbf{i})\}$ as summations of monomial realizations of certain Demazure crystals.
\end{abstract}

Key words: cluster variables; double Bruhat cells; crystal bases; monomial realizations, generalized minors

2010 Mathematics Subject Classification: 13F60; 81R50; 17B37

\section{Introduction}

As is well-known that theory of cluster algebras has been initiated by S. Fomin and A. Zelevinsky in the study of product expressions by $q$-commuting elements for upper global bases (= dual canonical bases). Crystal bases are obtained from global bases considering the parameter $q$ at 0 . Thus, we can guess that they should be deeply related each other at their origins.

Let $G$ be a simply connected simple algebraic group over $\mathbb{C}$ of rank $r$. Let $B$ and $B_{-}$be two opposite Borel subgroups in $G, N \subset B$ and $N_{-} \subset B_{-}$their unipotent radicals, $H:=B \cap B_{-}$ a maximal torus, and $W$ the associated Weyl group. In [1], it is shown that for $u, v \in W$ the coordinate ring $\mathbb{C}\left[G^{u, v}\right]$ of double Bruhat cell $G^{u, v}:=B u B \cap B_{-} v B_{-}$has the structure of an upper cluster algebra. The initial cluster variables of this upper cluster algebras are given as certain generalized minors on $G^{u, v}$.

In [11], the second author revealed the relations between some generalized minors and monomial realizations of crystal bases. A naive definition of monomial realizations of crystal bases is as follows (see Section 3 for the exact definitions): Let $\mathcal{Y}$ be the set of monomials in infinitely many variables (see Section 3, equation (3.2)). We shall define the crystal structures on $\mathcal{Y}$ associated with certain set of integers $p=\left(p_{i, j}\right)_{1 \leq i \neq j \leq r}$ and a Cartan matrix. And we can obtain a crystal for an irreducible module as a connected component of $\mathcal{Y}$. For example, for type $A_{4}$ and $p_{i, j}=1$ if $i<j$ and $p_{i, j}=0$ if $i>j$, we have the following crystal graph of the crystal $B\left(\Lambda_{3}\right)$, where $\Lambda_{3}$ is the $3^{\text {rd }}$ fundamental weight. The set of integers $p$ gives the cyclic sequence of indices

\footnotetext{
${ }^{\star}$ This paper is a contribution to the Special Issue on New Directions in Lie Theory. The full collection is available at http://www.emis.de/journals/SIGMA/LieTheory2014.html
} 
$\ldots 123412341234 \ldots$ and we associate variables $\left\{\tau_{j}\right\}$ as follows

$\begin{array}{cccccccccccccccccc}\ldots & 1 & 2 & 3 & 4 & 1 & 2 & 3 & 4 & 1 & 2 & 3 & 4 & 1 & 2 & 3 & 4 & \ldots \\ \ldots & \tau_{-4} & \tau_{-3} & \tau_{-2} & \tau_{-1} & \tau_{1} & \tau_{2} & \tau_{3} & \tau_{4} & \tau_{5} & \tau_{6} & \tau_{7} & & \tau_{8} & \tau_{9} & & \tau_{10} & \ldots\end{array}$

Note that the skip between $\tau_{7}$ and $\tau_{8}$ or $\tau_{9}$ and $\tau_{10}$ means no corresponding variable appears in the following crystal graph

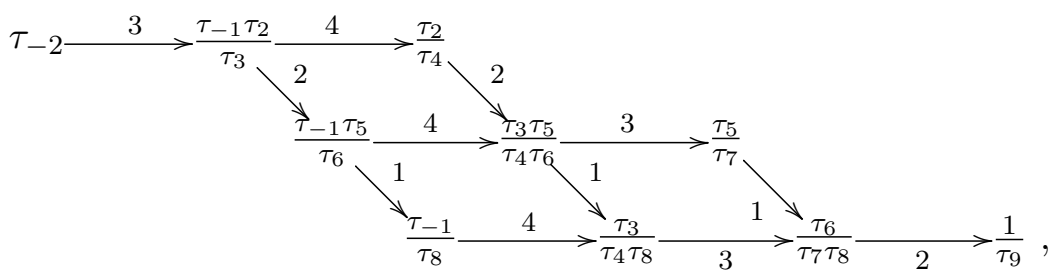

where the highest weight monomial $\tau_{-2}$ has a weight $\Lambda_{3}$ and the lowest weight monomial $\frac{1}{\tau_{9}}$ has a weight $-\Lambda_{2}$.

As stated above, the initial cluster variables on $G^{u, v}$ are expressed by generalized minors $\{\Delta(k ; \mathbf{i}) \mid 1 \leq k \leq l(u)+l(v)\}$, where $\mathbf{i}$ is the reduced expression of $(u, v) \in W \times W$. Now, as an example we consider the case $G=\mathrm{SL}_{5}(\mathbb{C})$ as above. Let $W=\mathfrak{S}_{5}=\left\langle s_{i} \mid 1 \leq i \leq 4\right\rangle$ be the symmetric group and set $u:=s_{1} s_{2} s_{3} s_{4} s_{1} s_{2} s_{3} s_{1} s_{2} s_{1}, v:=e$, and set a reduced word $\mathbf{i}$ for $u$ as $\mathbf{i}:=(1,2,3,4,1,2,3,1,2,1)$. We have for $x=\left(x_{i, j}\right) \in \mathrm{SL}_{5}(\mathbb{C})$

$$
\Delta(6 ; \mathbf{i})(x)=\left|\begin{array}{ll}
x_{31} & x_{32} \\
x_{41} & x_{42}
\end{array}\right|=x_{31} x_{42}-x_{32} x_{41}
$$

See Section 5 for the detailed explanation.

Now, let us consider the generalized minors on $L^{u, v}:=N u N \cap B_{-} v B_{-}$instead of $G^{u, v}$ since their difference is, indeed, only the factor from the torus part. We call $L^{u, v}$ reduced double Bruhat cell. For the above $u, v$, there exists a birational map $\mathbf{x}_{\mathbf{i}}^{L}:\left(\mathbb{C}^{\times}\right)^{10} \stackrel{\sim}{\longrightarrow} L^{u, v}$ given by

$$
\begin{aligned}
\mathbf{x}_{\mathbf{i}}^{L}\left(\tau_{1}, \ldots, \tau_{10}\right) \\
\quad=x_{-1}\left(\tau_{1}\right) x_{-2}\left(\tau_{2}\right) x_{-3}\left(\tau_{3}\right) x_{-4}\left(\tau_{4}\right) x_{-1}\left(\tau_{5}\right) x_{-2}\left(\tau_{6}\right) x_{-3}\left(\tau_{7}\right) x_{-1}\left(\tau_{8}\right) x_{-2}\left(\tau_{9}\right) x_{-1}\left(\tau_{10}\right) \\
\quad=\left(\begin{array}{ccccc}
\frac{1}{\tau_{1} \tau_{5} \tau_{8} \tau_{10}} & 0 & 0 & 0 & 0 \\
A & \frac{\tau_{1} \tau_{5} \tau_{8} \tau_{10}}{\tau_{2} \tau_{6} \tau_{9}} & 0 & 0 & 0 \\
B & C & \frac{\tau_{2} \tau_{6} \tau_{9}}{\tau_{3} \tau_{7}} & 0 & 0 \\
D & E & \frac{\tau_{3} \tau_{9}}{\tau_{4}}+\frac{\tau_{6} \tau_{9}}{\tau_{7}} & \frac{\tau_{3} \tau_{7}}{\tau_{4}} & 0 \\
1 & \tau_{10} & \tau_{9} & \tau_{7} & \tau_{4}
\end{array}\right)
\end{aligned}
$$

where

$$
\begin{aligned}
& A=\frac{\tau_{1} \tau_{5} \tau_{8}}{\tau_{2} \tau_{6} \tau_{9}}+\frac{\tau_{1} \tau_{5}}{\tau_{2} \tau_{6} \tau_{10}}+\frac{\tau_{1}}{\tau_{2} \tau_{8} \tau_{10}}+\frac{1}{\tau_{5} \tau_{8} \tau_{10}}, \\
& B=\frac{\tau_{2} \tau_{6}}{\tau_{3} \tau_{7}}+\frac{\tau_{2} \tau_{8}}{\tau_{3} \tau_{9}}+\frac{\tau_{5} \tau_{8}}{\tau_{6} \tau_{9}}+\frac{\tau_{2}}{\tau_{3} \tau_{10}}+\frac{\tau_{5}}{\tau_{6} \tau_{10}}+\frac{1}{\tau_{8} \tau_{10}}, \\
& C=\frac{\tau_{2} \tau_{6} \tau_{10}}{\tau_{3} \tau_{7}}+\frac{\tau_{2} \tau_{8} \tau_{10}}{\tau_{3} \tau_{9}}+\frac{\tau_{5} \tau_{8} \tau_{10}}{\tau_{6} \tau_{9}}, \\
& D=\frac{\tau_{3}}{\tau_{4}}+\frac{\tau_{6}}{\tau_{7}}+\frac{\tau_{8}}{\tau_{9}}+\frac{1}{\tau_{10}}, \quad E=\frac{\tau_{3} \tau_{10}}{\tau_{4}}+\frac{\tau_{6} \tau_{10}}{\tau_{7}}+\frac{\tau_{8} \tau_{10}}{\tau_{9}},
\end{aligned}
$$




$$
x_{-i}(t)=i^{\text {th }}\left(\begin{array}{cccc}
\ddots & & & \\
& t^{-1} & 0 & \\
& 1 & t & \\
& & & \ddots
\end{array}\right) .
$$

Therefore, by (1.2) and (1.3) we find

$$
\begin{aligned}
\Delta^{L}(6 ; \mathbf{i})(\tau) & :=\left(\Delta(6 ; \mathbf{i}) \circ x_{\mathbf{i}}^{L}\right)\left(\tau_{1}, \ldots, \tau_{10}\right)=\left|\begin{array}{cc}
B & C \\
D & E
\end{array}\right| \\
& =\frac{\tau_{2}}{\tau_{4}}+\frac{\tau_{3} \tau_{5}}{\tau_{4} \tau_{6}}+\frac{\tau_{5}}{\tau_{7}}+\frac{\tau_{3}}{\tau_{4} \tau_{8}}+\frac{\tau_{6}}{\tau_{7} \tau_{8}}+\frac{1}{\tau_{9}}
\end{aligned}
$$

Now, observing the crystal graph (1.1) and the Laurent polynomial (1.4), we realize that each term in (1.4) appears in (1.1) and they constitute so-called lower Demazure crystal associated with the element $u_{\leq 6} \in \mathfrak{S}_{5}[7]$.

Those facts motivate us to find a new linkage between the cluster variables on $L^{u, v} \subset G^{u, v}$ and the monomial realizations of crystals.

In this paper, we shall treat the case $G=\mathrm{SL}_{r+1}(\mathbb{C}), v=e$ and some special $u \in W=\mathfrak{S}_{r+1}$. More precisely, we treat an element $u \in W$ whose reduced word (Definition 2.1) can be written as a left factor of the standard longest word $(1,2,3, \ldots, r, 1,2,3, \ldots,(r-1), \ldots, 1,2,1)$ :

$$
u=s_{1} s_{2} \cdots s_{r} s_{1} \cdots s_{r-1} \cdots s_{1} \cdots s_{r-m+2} s_{1} \cdots s_{i_{n}}
$$

where $n:=l(u)$ is the length of $u$ and $1 \leq i_{n} \leq r-m+1$. And we treat (reduced) double Bruhat cells of the form $G^{u, e}:=B u B \cap B_{-}$and $L^{u, e}:=N u N \cap B_{-}$, where $B$ (resp. $B_{-}$) is the subgroup of upper (resp. lower) triangular matrices in $G=\mathrm{SL}_{r+1}(\mathbb{C})$. Then generalized minors are a part of classical minors (Definition 4.10). This case matches well to the Demazure crystals. In fact, we shall describe generalized minors in terms of summations over certain monomial realizations of Demazure crystals in the main result Theorem 5.6. For example, (1.4) shows that the generalized minor $\Delta^{L}(6 ; \mathbf{i})(\tau)$ is described in terms of summation over certain monomial realization of the Demazure crystal $B_{u \leq 6}^{-}\left(-\Lambda_{2}\right)$.

In forthcoming paper, we shall treat more general setting, like as, the Weyl group element $v \in W$ is non-identity or type $\mathrm{C}$. In these cases, the generalized minors are described also by monomial realizations of crystals.

\section{Factorization theorem for type A}

In this section, we shall introduce (reduced) double Bruhat cells $G^{u, v}, L^{u, v}$, and their properties in the case $G=\mathrm{SL}_{r+1}(\mathbb{C}), v=e$ and some special $u \in W$. In [2,3], these properties had been proven for simply connected, connected, semisimple complex algebraic groups and arbitrary $u, v \in W$.

For $l \in \mathbb{Z}_{>0}$, we set $[1, l]:=\{1,2,3, \ldots, l\}$.

\subsection{Double Bruhat cells}

Let $G=\mathrm{SL}_{r+1}(\mathbb{C})$ be the simple complex algebraic group of type $\mathrm{A}_{r}, B$ and $B_{-}$be two opposite Borel subgroups in $G$, that is, $B$ (resp. $B_{-}$) is the subgroup of upper (resp. lower) triangular matrices in $G=\mathrm{SL}_{r+1}(\mathbb{C})$. Let $N \subset B$ and $N_{-} \subset B_{-}$be their unipotent radicals, $H:=B \cap B_{-}$ a maximal torus, and $W:=\operatorname{Norm}_{G}(H) / H$ the Weyl group. In this case, Weyl group $W$ is isomorphic to the symmetric group $\mathfrak{S}_{r+1}$. 
We have two kinds of Bruhat decompositions of $G$ as follows

$$
G=\coprod_{u \in W} B u B=\coprod_{u \in W} B_{-} u B_{-} .
$$

Then, for $u, v \in W$, we define the double Bruhat cell $G^{u, v}$ as follows

$$
G^{u, v}:=B u B \cap B_{-} v B_{-} .
$$

This is biregularly isomorphic to a Zariski open subset of an affine space of dimension $r+l(u)+$ $l(v)[3$, Theorem 1.1].

We also define the reduced double Bruhat cell $L^{u, v}$ as follows

$$
L^{u, v}:=N u N \cap B_{-} v B_{-} \subset G^{u, v} .
$$

As is similar to the case $G^{u, v}, L^{u, v}$ is biregularly isomorphic to a Zariski open subset of an affine space of dimension $l(u)+l(v)[2$, Proposition 4.4].

Definition 2.1. Let $u=s_{i_{1}} \cdots s_{i_{n}}$ be a reduced expression of $u \in W, i_{1}, \ldots, i_{n} \in[1, r]$. Then the finite sequence

$$
\mathbf{i}:=\left(i_{1}, \ldots, i_{n}\right)
$$

is called reduced word $\mathbf{i}$ for $u$.

In this paper, we treat (reduced) double Bruhat cells of the form $G^{u, e}:=B u B \cap B_{-}$and $L^{u, e}:=N u N \cap B_{-}$, where $u \in W$ is an element whose reduced word can be written as a left factor of $(1,2,3, \ldots, r, 1,2,3, \ldots,(r-1), \ldots, 1,2,1)$ :

$$
u=s_{1} s_{2} \cdots s_{r} s_{1} \cdots s_{r-1} \cdots s_{1} \cdots s_{r-m+2} s_{1} \cdots s_{i_{n}}
$$

where $n:=l(u)$ is the length of $u$ and $1 \leq i_{n} \leq r-m+1$. Let $\mathbf{i}$ be a reduced word of $u$ :

$$
\mathbf{i}=(\underbrace{1, \ldots, r}_{1^{\text {st }} \text { cycle }}, \underbrace{1, \ldots,(r-1)}_{2^{\text {nd }} \text { cycle }}, \ldots, \underbrace{1, \ldots,(r-m+2)}_{(m-1)^{\text {th }} \text { cycle }}, \underbrace{1, \ldots, i_{n}}_{m^{\text {th }} \text { cycle }}) .
$$

Note that $(1,2,3, \ldots, r, 1,2,3, \ldots,(r-1), \ldots, 1,2,1)$ is a reduced word of the longest element in $W$.

\subsection{Factorization theorem for type A}

In this subsection, we shall introduce the isomorphisms between double Bruhat cell $G^{u, e}$ and $H \times\left(\mathbb{C}^{\times}\right)^{l(u)}$, and between $L^{u, e}$ and $\left(\mathbb{C}^{\times}\right)^{l(u)}$. As in the previous section, we consider the case $G:=\mathrm{SL}_{r+1}(\mathbb{C})$. We set $\mathfrak{g}:=\operatorname{Lie}(G)$ with the Cartan decomposition $\mathfrak{g}=\mathfrak{n}-\oplus \mathfrak{h} \oplus \mathfrak{n}$. Let $e_{i}, f_{i}$ $(i \in[1, r])$ be the generators of $\mathfrak{n}, \mathfrak{n}_{-}$. For $i \in[1, r]$ and $t \in \mathbb{C}$, we set $x_{i}(t):=\exp \left(t e_{i}\right)$, $y_{i}:=\exp \left(t f_{i}\right)$. Let $\varphi_{i}: \mathrm{SL}_{2}(\mathbb{C}) \rightarrow G$ be the canonical embedding corresponding to each simple root $\alpha_{i}$. Then we have

$$
x_{i}(t)=\varphi_{i}\left(\begin{array}{cc}
1 & t \\
0 & 1
\end{array}\right), \quad y_{i}(t)=\varphi_{i}\left(\begin{array}{ll}
1 & 0 \\
t & 1
\end{array}\right) .
$$

We can express $x_{i}(t), y_{i}(t)$, as the following matrices

$$
x_{i}(t)=i^{\text {th }}\left(\begin{array}{llll}
\ddots & & & \\
& 1 & t & \\
& 0 & 1 & \\
& & & \ddots
\end{array}\right), \quad y_{i}(t)=i^{\text {th }}\left(\begin{array}{llll}
\ddots & & & \\
& 1 & 0 & \\
& t & 1 & \\
& & & \ddots
\end{array}\right) .
$$


For a reduced word $\mathbf{i}=\left(i_{1}, i_{2}, \ldots, i_{n}\right)$, we define a map $x_{\mathbf{i}}^{G}: H \times \mathbb{C}^{n} \rightarrow G$ as

$$
x_{\mathbf{i}}^{G}\left(a ; t_{1}, \ldots t_{n}\right):=a \cdot y_{i_{1}}\left(t_{1}\right) y_{i_{2}}\left(t_{2}\right) \cdots y_{i_{n}}\left(t_{n}\right) .
$$

Theorem 2.2 ([3, Theorem 1.2]). We set $u \in W$ and its reduced word $\mathbf{i}$ as in (2.1) and (2.2). The map $x_{\mathrm{i}}^{G}$ defined above can be restricted to a biregular isomorphism between $H \times\left(\mathbb{C}^{\times}\right)^{l(u)}$ and a Zariski open subset of $G^{u, e}$.

Next, for $i \in[1, r]$ and $t \in \mathbb{C}^{\times}$, we define as follows

$$
\alpha_{i}^{\vee}(t):=\varphi_{i}\left(\begin{array}{cc}
t & 0 \\
0 & t^{-1}
\end{array}\right), \quad x_{-i}(t):=y_{i}(t) \alpha_{i}^{\vee}\left(t^{-1}\right)=\varphi_{i}\left(\begin{array}{cc}
t^{-1} & 0 \\
1 & t
\end{array}\right) .
$$

We can express $x_{-i}(t)$ and $\alpha_{i}^{\vee}(t)$ as the following matrices

$$
x_{-i}(t)={ }^{\text {th }}\left(\begin{array}{cccc}
\ddots & & & \\
& t^{-1} & 0 & \\
& 1 & t & \\
& & & \ddots
\end{array}\right), \quad \alpha_{i}^{\vee}(t)=\operatorname{diag}\left(1, \ldots, 1, \stackrel{i}{\left.\stackrel{i}{*}, t^{-1}, 1, \ldots, 1\right) .}\right.
$$

For $\mathbf{i}=\left(i_{1}, \ldots, i_{n}\right)\left(i_{1}, \ldots, i_{n} \in[1, r]\right)$, we define a map $x_{\mathbf{i}}^{L}: \mathbb{C}^{n} \rightarrow G$ as

$$
x_{\mathbf{i}}^{L}\left(t_{1}, \ldots, t_{n}\right):=x_{-i_{1}}\left(t_{1}\right) \cdots x_{-i_{n}}\left(t_{n}\right) .
$$

We have the following theorem which is similar to the previous one.

Theorem 2.3 ([2, Proposition 4.5]). We set $u \in W$ and its reduced word $\mathbf{i}$ as in (2.1) and (2.2). The map $x_{\mathbf{i}}^{L}$ defined above can be restricted to a biregular isomorphism between $\left(\mathbb{C}^{\times}\right)^{l(u)}$ and a Zariski open subset of $L^{u, e}$.

Finally, we define a map $\bar{x}_{\mathbf{i}}^{G}: H \times\left(\mathbb{C}^{\times}\right)^{n} \rightarrow G^{u, v}$ as

$$
\bar{x}_{\mathbf{i}}^{G}\left(a ; t_{1}, \ldots, t_{n}\right)=a x_{\mathbf{i}}^{L}\left(t_{1}, \ldots, t_{n}\right) .
$$

Proposition 2.4. In the above setting, the map $\bar{x}_{\mathbf{i}}^{G}$ is a biregular isomorphism between $H \times$ $\left(\mathbb{C}^{\times}\right)^{n}$ and a Zariski open subset of $G^{u, e}$.

Proof. We set $l_{0}:=0, l_{1}:=r, l_{2}:=r+(r-1), \ldots, l_{m}:=r+(r-1)+\cdots+(r-m+1)$. We define a map $\phi: H \times\left(\mathbb{C}^{\times}\right)^{n} \rightarrow H \times\left(\mathbb{C}^{\times}\right)^{n}, \mathbf{t}=\left(a ; t_{1}, \ldots, t_{n}\right) \mapsto\left(a(\mathbf{t}) ; \tau_{1}(\mathbf{t}), \ldots, \tau_{n}(\mathbf{t})\right)$ as

$$
\begin{aligned}
& a(\mathbf{t})=a \cdot \underbrace{\alpha_{1}^{\vee}\left(t_{1}\right)^{-1} \cdots \alpha_{r}^{\vee}\left(t_{r}\right)^{-1}}_{1^{\text {st }} \text { cycle }} \cdots \underbrace{\alpha_{1}^{\vee}\left(t_{l_{m-1}+1}\right)^{-1} \cdots \alpha_{i_{n}}^{\vee}\left(t_{l_{m-1}+i_{n}}\right)^{-1}}_{m^{\text {th }} \text { cycle }} \\
& \tau_{l_{s}+j}(\mathbf{t})=\frac{\left(t_{l_{s+1}+j-1} t_{l_{s+2}+j-1} \cdots t_{l_{m-1}+j-1}\right)\left(t_{l_{s}+j+1} t_{l_{s+1}+j+1} \cdots t_{l_{m-1}+j+1}\right)}{t_{l_{s}+j}\left(t_{l_{s+1}+j} \cdots t_{l_{m-1}+j}\right)^{2}}
\end{aligned}
$$

where in $(2.5)$, if $\mathbf{i}$ does not include $j$ (resp. $j+1, j-1$ ) in $\zeta^{\text {th }}$ cycle then we set $t_{l_{\zeta-1}+j}=1$ (resp. $t_{l_{\zeta-1}+j+1}=1, t_{l_{\zeta-1}+j-1}=1$ ). This is a biregular isomorphism.

Let us prove

$$
\bar{x}_{\mathbf{i}}^{G}\left(a ; t_{1}, \ldots, t_{n}\right)=\left(x_{\mathbf{i}}^{G} \circ \phi\right)\left(a ; t_{1}, \ldots, t_{n}\right),
$$

which implies that $\bar{x}_{\mathbf{i}}^{G}: H \times\left(\mathbb{C}^{\times}\right)^{n} \rightarrow G^{u, e}$ is a biregular isomorphism by Theorem 2.2 . 
First, we can verify the following relations by the explicit forms $(2.3),(2.4)$ and direct calculations:

$$
\alpha_{i}^{\vee}(c)^{-1} y_{j}(t)= \begin{cases}y_{i}\left(c^{2} t\right) \alpha_{i}^{\vee}(c)^{-1} & \text { if } \quad i=j \\ y_{j}\left(c^{-1} t\right) \alpha_{i}^{\vee}(c)^{-1} & \text { if } \quad|i-j|=1 \\ y_{j}(t) \alpha_{i}^{\vee}(c)^{-1} & \text { otherwise, }\end{cases}
$$

for $1 \leq i, j \leq r$ and $c, t \in \mathbb{C}^{\times}$.

On the other hand, we obtain

$$
\begin{aligned}
& \left(x_{\mathbf{i}}^{G} \circ \phi\right)\left(a ; t_{1}, \ldots, t_{n}\right)=a \times \alpha_{1}^{\vee}\left(t_{1}\right)^{-1} \cdots \alpha_{r}^{\vee}\left(t_{r}\right)^{-1} \cdots \alpha_{1}^{\vee}\left(t_{l_{m-1}+1}\right)^{-1} \cdots \alpha_{i_{n}}^{\vee}\left(t_{l_{m-1}+i_{n}}\right)^{-1} \\
& \quad \times y_{1}\left(\tau_{1}(\mathbf{t})\right) y_{2}\left(\tau_{2}(\mathbf{t})\right) \cdots y_{r}\left(\tau_{r}(\mathbf{t})\right) \cdots y_{1}\left(\tau_{l_{m-1}+1}(\mathbf{t})\right) \cdots y_{i_{n}}\left(\tau_{l_{m-1}+i_{n}}(\mathbf{t})\right) .
\end{aligned}
$$

For each $s$ and $j$, let us move $\alpha_{j}^{\vee}\left(t_{l_{s}+j}\right)^{-1}, \alpha_{j+1}^{\vee}\left(t_{l_{s}+j+1}\right)^{-1}, \ldots, \alpha_{i_{n}}^{\vee}\left(t_{l_{m-1}+i_{n}}\right)^{-1}$ to the right of $y_{j}\left(\tau_{l_{s}+j}(\mathbf{t})\right)$ by using the relations (2.6). For example,

$$
\begin{aligned}
\alpha_{j}^{\vee}\left(t_{l_{s}+j}\right)^{-1} \cdots \alpha_{j-1}^{\vee}\left(t_{l_{m-1}+j-1}\right)^{-1} \alpha_{j}^{\vee}\left(t_{l_{m-1}+j}\right)^{-1} & \\
& \times \alpha_{j+1}^{\vee}\left(t_{l_{m-1}+j+1}\right)^{-1} \cdots \alpha_{i_{n}}^{\vee}\left(t_{l_{m-1}+i_{n}}\right)^{-1} y_{j}\left(\tau_{l_{s}+j}(\mathbf{t})\right) \\
= & \alpha_{j}^{\vee}\left(t_{l_{s}+j}\right)^{-1} \cdots y_{j}\left(\frac{t_{l_{m-1}+j}^{2}}{t_{l_{m-1}+j-1} t_{l_{m-1}+j+1}} \tau_{l_{s}+j}(\mathbf{t})\right) \alpha_{j-1}^{\vee}\left(t_{l_{m-1}+j-1}\right)^{-1} \\
& \times \alpha_{j}^{\vee}\left(t_{l_{m-1}+j}\right)^{-1} \alpha_{j+1}^{\vee}\left(t_{l_{m-1}+j+1}\right)^{-1} \cdots \alpha_{i_{n}}^{\vee}\left(t_{l_{m-1}+i_{n}}\right)^{-1} .
\end{aligned}
$$

Repeating this argument, we have

$$
=y_{j}\left(\frac{\left(t_{l_{s}+j} \cdots t_{l_{m-1}+j}\right)^{2}}{\left(t_{l_{s}+j-1} \cdots t_{l_{m-1}+j-1}\right)\left(t_{l_{s}+j+1} \cdots t_{l_{m-1}+j+1}\right)} \tau_{l_{s}+j}(\mathbf{t})\right) \alpha_{j}^{\vee}\left(t_{l_{s}+j}\right)^{-1} \cdots \alpha_{i_{n}}^{\vee}\left(t_{l_{m-1}+i_{n}}\right)^{-1} .
$$

Note that $\frac{\left(t_{l_{s}+j} \ldots t_{l_{m-1}+j}\right)^{2}}{\left(t_{l_{s}+j-1} \cdots t_{l_{m-1}+j-1}\right)\left(t_{l_{s}+j+1} \cdots t_{l_{m-1}+j+1}\right)} \tau_{l_{s}+j}(\mathbf{t})=t_{l_{s}+j}$. By $(2.7)$, we have

$$
\begin{aligned}
\left(x_{\mathbf{i}}^{G} \circ \phi\right) & \left(a ; t_{1}, \ldots, t_{n}\right)=a \cdot y_{1}\left(t_{1}\right) \alpha_{1}^{\vee}\left(t_{1}\right)^{-1} \cdots y_{r}\left(t_{r}\right) \alpha_{r}^{\vee}\left(t_{r}\right)^{-1} \ldots \\
& \times y_{1}\left(t_{l_{m-1}+1}\right) \alpha_{1}^{\vee}\left(t_{l_{m-1}+1}\right)^{-1} \cdots y_{i_{n}}\left(t_{l_{m-1}+i_{n}}\right) \alpha_{i_{n}}^{\vee}\left(t_{l_{m-1}+i_{n}}\right)^{-1} \\
= & a \cdot x_{-1}\left(t_{1}\right) \cdots x_{-r}\left(t_{r}\right) \cdots x_{-1}\left(t_{l_{m-1}+1}\right) \cdots x_{-i_{n}}\left(t_{l_{m-1}+i_{n}}\right)=\bar{x}_{\mathbf{i}}^{G}\left(a ; t_{1}, \ldots, t_{n}\right) .
\end{aligned}
$$

\section{Monomial realizations of crystal bases}

In this section, we review the monomial realizations of crystals $[6,8,10]$. Let $I:=\{1,2, \ldots, r\}$ be a finite index set.

\subsection{Monomial realizations of crystal bases for type A}

Definition 3.1. Let $A=\left(a_{i j}\right)_{i, j \in I}$ be the Cartan matrix of type $\mathrm{A}_{r}: A=\left(a_{i j}\right)_{i, j \in I}$ is defined as

$$
a_{i j}= \begin{cases}2 & \text { if } \quad i=j \\ -1 & \text { if } \quad|i-j|=1 \\ 0 & \text { otherwise }\end{cases}
$$

Let $\Pi=\left\{\alpha_{i} \mid i \in I\right\}$ (resp. $\Pi^{\vee}=\left\{h_{i} \mid i \in I\right\}$ ) be the set of simple roots (resp. co-roots), and $P$ be the weight lattice. A crystal associated with the Cartan matrix $A$ is a set $B$ together with the maps wt $: B \rightarrow P, \tilde{e}_{i}, \tilde{f}_{i}: B \cup\{0\} \rightarrow B \cup\{0\}$ and $\varepsilon_{i}, \varphi_{i}: B \rightarrow \mathbb{Z} \cup\{-\infty\}, i \in I$, satisfying the following properties: For $b \in B, i \in I$, 
(i) $\varphi_{i}(b)-\varepsilon_{i}(b)=\left\langle h_{i}, \mathrm{wt}(b)\right\rangle$,

(ii) $\operatorname{wt}\left(\tilde{e}_{i} b\right)=\operatorname{wt}(b)+\alpha_{i}$, if $\tilde{e}_{i} b \in B$,

(iii) $\operatorname{wt}\left(\tilde{f}_{i} b\right)=\operatorname{wt}(b)-\alpha_{i}$, if $\tilde{f}_{i} b \in B$,

(iv) $\varepsilon_{i}\left(\tilde{e}_{i} b\right)=\varepsilon_{i}(b)-1, \varphi_{i}\left(\tilde{e}_{i} b\right)=\varphi_{i}(b)+1$ if $\tilde{e}_{i} b \in B$,

(v) $\varepsilon_{i}\left(\tilde{f}_{i} b\right)=\varepsilon_{i}(b)+1, \varphi_{i}\left(\tilde{f}_{i} b\right)=\varphi_{i}(b)-1$ if $\tilde{f}_{i} b \in B$,

(vi) $\tilde{f}_{i} b=b^{\prime} \Leftrightarrow b=\tilde{e}_{i} b^{\prime}$, if $b, b^{\prime} \in B$,

(vii) $\varphi_{i}(b)=-\infty, b \in B, \Rightarrow \tilde{e}_{i} b=\tilde{f}_{i} b=0$.

Let $U_{q}(\mathfrak{g})$ be the universal enveloping algebra associated with the Cartan matrix $A$ in (3.1), and $\mathfrak{g}=\mathfrak{s l}_{r+1}(\mathbb{C})$. Let $B^{+}(\lambda)$ (resp. $B^{-}(\lambda)$ ) be the crystal base of the $U_{q}(\mathfrak{g}$ )-highest (resp. lowest) weight module [5,9]. Note that $B^{+}(\lambda)=B^{-}\left(w_{0} \lambda\right)$, where $w_{0}$ is the longest element of $W$. In particular, in the case $\lambda=M \Lambda_{d}, M \in \mathbb{Z}_{>0}$, we have

$$
B^{+}\left(M \Lambda_{d}\right)=B^{-}\left(-M \Lambda_{r-d+1}\right) .
$$

Let us introduce monomial realizations which realize each element of $B^{ \pm}(\lambda)$ as a certain Laurent monomial.

First, we define a set of integers $p=\left(p_{j, i}\right)_{j, i \in I, j \neq i}$ such that

$$
p_{j, i}= \begin{cases}1 & \text { if } \quad j<i \\ 0 & \text { if } \quad i<j\end{cases}
$$

Second, for doubly-indexed variables $\left\{Y_{s, i} \mid i \in I, s \in \mathbb{Z}\right\}$, we define the set of monomials

$$
\mathcal{Y}:=\left\{Y=\prod_{s \in \mathbb{Z}, i \in I} Y_{s, i}^{\zeta_{s, i}} \mid \zeta_{s, i} \in \mathbb{Z}, \zeta_{s, i}=0 \text { except for finitely many }(s, i)\right\} .
$$

Finally, we define maps wt $: \mathcal{Y} \rightarrow P, \varepsilon_{i}, \varphi_{i}: \mathcal{Y} \rightarrow \mathbb{Z}, i \in I$. For $Y=\prod_{s \in \mathbb{Z}, i \in I} Y_{s, i}^{\zeta_{s, i}} \in \mathcal{Y}$,

$$
\operatorname{wt}(Y):=\sum_{i, s} \zeta_{s, i} \Lambda_{i}, \quad \varphi_{i}(Y):=\max \left\{\sum_{k \leq s} \zeta_{k, i} \mid s \in \mathbb{Z}\right\}, \quad \varepsilon_{i}(Y):=\varphi_{i}(Y)-\operatorname{wt}(Y)\left(h_{i}\right) .
$$

We set

$$
A_{s, i}:=Y_{s, i} Y_{s+1, i} \prod_{j \neq i} Y_{s+p_{j, i}, j}^{a_{j, i}}= \begin{cases}\frac{Y_{s, 1} Y_{s+1,1}}{Y_{s, 2}} & \text { if } \quad i=1, \\ \frac{Y_{s, i} Y_{s+1, i}}{Y_{s, i+1} Y_{s+1, i-1}} & \text { if } \quad 2 \leq i \leq r-1, \\ \frac{Y_{s, r} Y_{s+1, r}}{Y_{s+1, r-1}} & \text { if } \quad i=r,\end{cases}
$$

and define the Kashiwara operators as follows

$$
\tilde{f}_{i} Y=\left\{\begin{array}{lll}
A_{n_{f_{i}}, i}^{-1} Y & \text { if } & \varphi_{i}(Y)>0, \\
0 & \text { if } & \varphi_{i}(Y)=0,
\end{array} \quad \tilde{e}_{i} Y=\left\{\begin{array}{lll}
A_{n_{e_{i}}, i} Y & \text { if } & \varepsilon_{i}(Y)>0 \\
0 & \text { if } & \varepsilon_{i}(Y)=0
\end{array}\right.\right.
$$

where

$$
n_{f_{i}}:=\min \left\{n \mid \varphi_{i}(Y)=\sum_{k \leq n} \zeta_{k, i}\right\}, \quad n_{e_{i}}:=\max \left\{n \mid \varphi_{i}(Y)=\sum_{k \leq n} \zeta_{k, i}\right\} .
$$

Then the following theorem holds: 
Theorem $3.2([8,10])$.

(i) For the set $p=\left(p_{j, i}\right)$ as above, $\left(\mathcal{Y}, w t, \varphi_{i}, \varepsilon_{i}, \tilde{f}_{i}, \tilde{e}_{i}\right)_{i \in I}$ is a crystal. When we emphasize $p$, we write $\mathcal{Y}$ as $\mathcal{Y}(p)$.

(ii) If a monomial $Y \in \mathcal{Y}(p)$ satisfies $\varepsilon_{i}(Y)=0$ (resp. $\left.\varphi_{i}(Y)=0\right)$ for all $i \in I$, then the connected component containing $Y$ is isomorphic to $B^{+}(w t(Y))\left(\right.$ resp. $\left.B^{-}(w t(Y))\right)$.

Definition 3.3. Let $Y \in \mathcal{Y}(p)$ be a monomial and let $\mathbb{B}$ be the unique connected component in $\mathcal{Y}(p)$ including $Y$. Suppose that $\lambda$ is the highest (resp. lowest) weight of $\mathbb{B}$. We denote the embedding

$$
\mu_{Y}: B^{+}(\lambda) \hookrightarrow \mathbb{B} \subset \mathcal{Y}(p) \quad\left(\text { resp. } \mu_{Y}: B^{-}(\lambda) \hookrightarrow \mathbb{B}\right) .
$$

Note that if $Y$ and $Y^{\prime}$ are in the same component then $\mu_{Y}=\mu_{Y^{\prime}}$.

Remark 3.4. The actions of $\tilde{e}_{i}$ and $\tilde{f}_{i}$ on $Y$ are determined by $\operatorname{wt}(Y)\left(h_{i}\right), \varphi_{i}(Y)$ and $\varepsilon_{i}(Y)$, which are determined by the factors $Y_{s, i}^{ \pm 1}, s \in \mathbb{Z}$. Thus, when we consider the actions of $\tilde{e}_{i}$ and $\tilde{f}_{i}$, we need to see the factors $\left\{Y_{s, i}^{ \pm 1}\right\}_{s \in \mathbb{Z}}$ only.

Example 3.5. For $\lambda=\beta \Lambda_{d}$ (resp. $\left.\lambda=-\beta \Lambda_{d}\right), \beta \in \mathbb{Z}_{>0}, d \in I$, we can embed $B^{+}(\lambda)$ (resp. $\left.B^{-}(\lambda)\right)$ in $\mathcal{Y}$ as a crystal by

$$
v_{\lambda} \mapsto Y_{\beta+\gamma, d} Y_{\beta-1+\gamma, d} \cdots Y_{1+\gamma, d}, \quad\left(\text { resp. } v_{\lambda} \mapsto \frac{1}{Y_{\beta+\gamma, d} Y_{\beta-1+\gamma, d} \cdots Y_{1+\gamma, d}}\right)
$$

where $v_{\lambda}$ is the highest (resp. lowest) weight vector of $B^{+}(\lambda)$ (resp. $B^{-}(\lambda)$ ), and $\gamma$ is an arbitrary integer. For $Y^{+}:=Y_{\beta+\gamma, d} Y_{\beta-1+\gamma, d} \cdots Y_{1+\gamma, d}\left(\right.$ resp. $\left.Y^{-}:=\frac{1}{Y_{\beta+\gamma, d} Y_{\beta-1+\gamma, d} \cdots Y_{1+\gamma, d}}\right), \mu_{Y^{+}}$ (resp. $\mu_{Y^{-}}$) denotes the embedding in Definition 3.3. Then $Y^{+}$(resp. $Y^{-}$) is the highest (resp. lowest) weight vector in $\mu_{Y^{+}}\left(B^{+}(\lambda)\right)$ (resp. $\left.\mu_{Y^{-}}\left(B^{-}(\lambda)\right)\right)$.

We set $l_{0}:=0, l_{1}:=r, l_{2}:=r+(r-1), \ldots, l_{s}:=r+(r-1)+\cdots+(r-s+1), \ldots$, $l_{r}:=r+(r-1)+\cdots+2+1$ and changing the variables $Y_{s, j}$ to $\tau_{l_{s}+j}, 1 \leq j \leq r-s$. For $s<0$, we transform the variables $Y_{s, j}$ to $\tau_{-(r+1-j)}, 1 \leq j \leq r$,

$$
\begin{array}{ccccccccccccc}
\ldots & r & 1 & \ldots & r-1 & r & 1 & \ldots & r-1 & r & 1 & 2 & \ldots \\
\ldots & \tau_{-1} & \tau_{l_{0}+1} & \ldots & \tau_{l_{0}+r-1} & \tau_{l_{0}+r} & \tau_{l_{1}+1} & \ldots & \tau_{l_{1}+r-1} & & \tau_{l_{2}+1} & \tau_{l_{2}+2} & \ldots
\end{array}
$$

Remark 3.6. In the above setting, the variables $\left\{Y_{s, j} \mid r-s<j\right\}$ do not correspond to any variables in $\tau$. As we have seen in (1.1), these variables do not appear in the crystal base which we treat in this paper. In other words, we only need variables associated with

$$
\mathbf{j}=(1, \ldots, r, 1, \ldots, r-1, \ldots, 1,2,1),
$$

which coincides with a specific reduced word of the longest element of $W$.

Remark 3.7. For the variables $\tau_{l_{s}+0}, \tau_{l_{s}+r+1}(0 \leq s \leq m-1)$ we understand

$$
\tau_{l_{s}+0}=\tau_{l_{s}+r+1}=1 .
$$

For example, if $i=1$ then

$$
\tau_{l_{s}+i-1}=1 .
$$


Example 3.8. Let us consider the action of $\tilde{e}_{1}$ on the monomial $\frac{1}{\tau_{l_{r-1}+1}}$. Following the method in Section 3.1, we have $\operatorname{wt}\left(\frac{1}{\tau_{l_{r-1}+1}}\right)=-\Lambda_{1}, \varphi_{1}\left(\frac{1}{\tau_{l_{r-1}+1}}\right)=0, \varepsilon_{1}\left(\frac{1}{\tau_{l_{r-1}+1}}\right)=\varphi_{1}\left(\frac{1}{\tau_{l_{r-1}+1}}\right)-$ $\operatorname{wt}\left(\frac{1}{\tau_{l_{r-1}+1}}\right)\left(h_{1}\right)=1$, and $n_{e_{1}}=r-2$. Thus, since we have $A_{r-2,1}=\tau_{l_{r-2}+1} \tau_{l_{r-1}+1} \tau_{l_{r-2}+p_{2,1}+2}^{a_{2,1}}=$ $\frac{\tau_{l_{r-2}+1} \tau_{l_{r-1}+1}}{\tau_{l_{r-2}+2}}$, we get

$$
\tilde{e}_{1} \frac{1}{\tau_{l_{r-1}+1}}=A_{r-2,1} \frac{1}{\tau_{l_{r-1}+1}}=\frac{\tau_{l_{r-2}+1}}{\tau_{l_{r-2}+2}} .
$$

Similarly, we have

$$
\begin{aligned}
& \tilde{e}_{2} \tilde{e}_{1} \frac{1}{\tau_{l_{r-1}+1}}=A_{r-3,2} \frac{\tau_{l_{r-2}+1}}{\tau_{l_{r-2}+2}}=\frac{\tau_{l_{r-3}+2}}{\tau_{l_{r-3}+3}}, \quad A_{r-3,2}=\frac{\tau_{l_{r-3}+2} \tau_{l_{r-2}+2}}{\tau_{l_{r-3}+3} \tau_{l_{r-2}+1}} \\
& \tilde{e}_{3} \tilde{e}_{2} \tilde{e}_{1} \frac{1}{\tau_{l_{r-1}+1}}=A_{r-4,3} \frac{\tau_{l_{r-3}+2}}{\tau_{l_{r-3}+3}}=\frac{\tau_{l_{r-4}+3}}{\tau_{l_{r-4}+4}}, \quad A_{r-4,3}=\frac{\tau_{l_{r-4}+3} \tau_{l_{r-3}+3}}{\tau_{l_{r-4}+4} \tau_{l_{r-3}+2}} .
\end{aligned}
$$

Applying $\tilde{e}_{i}$ repeatedly, we obtain

$$
\tilde{e}_{k} \cdots \tilde{e}_{2} \tilde{e}_{1} \frac{1}{\tau_{l_{r-1}+1}}=A_{r-1-k, k} \tilde{e}_{k-1} \cdots \tilde{e}_{2} \tilde{e}_{1} \frac{1}{\tau_{l_{r-1}+1}}=\frac{\tau_{l_{r-1-k}+k}}{\tau_{l_{r-1-k}+k+1}}, \quad k=1, \ldots, r
$$

where, $A_{r-1-k, k}=\frac{\tau_{l_{r-1-k}+k} \tau_{l_{r-k}+k}}{\tau_{l_{r-1-k}+k+1} \tau_{l_{r-k}+k-1}}, \tau_{l_{-1}+r}=r, \tau_{l_{-1}+r+1}:=1$. For $i \in I$, we have $\varphi_{i}\left(\frac{1}{\tau_{l_{r-1}+1}}\right)=0$. Hence $\tilde{f}_{i}\left(\frac{1}{\tau_{l_{r-1}+1}}\right)=0$.

Example 3.9. For a given $i \in I$ and $Y=\prod_{s \in \mathbb{Z}} \tau_{l_{s}+i}^{\zeta_{s, i}}$, we define $\nu_{Y}(n):=\sum_{s \leq n} \zeta_{s, i}$.

For $j \in \mathbb{Z}_{>0}$, we set

$$
Y=\frac{1}{\tau_{l_{q_{1}}+i} \tau_{l_{q_{2}}+i} \cdots \tau_{l_{q_{j}}+i}}, \quad 0 \leq q_{1}<q_{2}<\cdots<q_{j} \leq r-1 .
$$

First, let us calculate $n_{e_{i}}(3.4)$. We obtain $\operatorname{wt}(Y)=-j \Lambda_{i}$ and

$$
\begin{aligned}
& \nu_{Y}(n)=0 \quad \text { for } \quad n<0, \\
& \nu_{Y}(0)=\nu_{Y}(1)=\cdots=\nu_{Y}\left(q_{1}-1\right)=0, \quad \nu_{Y}\left(q_{1}\right)=\nu_{Y}\left(q_{1}+1\right)=\cdots=\nu_{Y}\left(q_{2}-1\right)=-1, \\
& \nu_{Y}\left(q_{2}\right)=\nu_{Y}\left(q_{2}+1\right)=\cdots=\nu_{Y}\left(q_{3}-1\right)=-2, \quad \nu_{Y}\left(q_{3}\right)=\cdots=\nu_{Y}\left(q_{4}-1\right)=-3, \quad \cdots
\end{aligned}
$$

Thus, we get $\varphi_{i}(Y)=\max \left\{\nu_{Y}(n) \mid n \in \mathbb{Z}\right\}=0$ and

$$
n_{e_{i}}=\max \left\{n \mid \nu_{Y}(n)=0\right\}=q_{1}-1 \text {. }
$$

Next, since $\operatorname{wt}(Y)\left(h_{i}\right)=-j$, we have $\varepsilon_{i}(Y)=\varphi_{i}(Y)-\operatorname{wt}(Y)\left(h_{i}\right)=j>0$. Therefore,

$$
\tilde{e}_{i} Y=A_{q_{1}-1, i} Y=\frac{\tau_{l_{q_{1}-1}+i}}{\tau_{l_{q_{1}-1}+i+1} \tau_{l_{q_{1}}+i-1} \tau_{l_{q_{2}}+i} \cdots \tau_{l_{q_{j}}+i}}, \quad A_{q_{1}-1, i}=\frac{\tau_{l_{q_{1}-1}+i} \tau_{l_{q_{1}}+i}}{\tau_{l_{q_{1}-1}+i+1} \tau_{l_{q_{1}}+i-1}} .
$$

Similarly, for $k=1,2, \ldots, j$, we get

$$
\tilde{e}_{i}^{k} Y=A_{q_{k}-1, i} \cdots A_{q_{2}-1, i} A_{q_{1}-1, i} Y=\prod_{s=1}^{k}\left(\frac{\tau_{l_{q_{k}-1}+i}}{\tau_{l_{q_{k}-1}+i+1} \tau_{l_{q_{k}}+i-1}}\right) \frac{1}{\tau_{l_{q_{k+1}}+i} \cdots \tau_{l_{j}+i}} .
$$




\subsection{Demazure crystal}

For $w \in W$, let us define an upper Demazure crystal $B_{w}^{+}(\lambda)$. This is a subset of the crystal $B^{+}(\lambda)$ defined as follows.

Definition 3.10. Let $u_{\lambda}$ be the highest weight vector of $B^{+}(\lambda)$. For the identity element $e$ of $W$, we set $B_{e}^{+}(\lambda):=\left\{u_{\lambda}\right\}$. For $w \in W$, if $s_{i} w<w$,

$$
B_{w}^{+}(\lambda):=\left\{\tilde{f}_{i}^{k} b \mid k \geq 0, b \in B_{s_{i} w}^{+}(\lambda), \tilde{e}_{i} b=0\right\} \backslash\{0\} .
$$

Similarly, we define a lower Demazure crystal $B_{w}^{-}(\lambda)$ as follows.

Definition 3.11. Let $v_{\lambda}$ be the lowest weight vector of $B^{-}(\lambda)$. We set $B_{e}^{-}(\lambda):=\left\{v_{\lambda}\right\}$. For $w \in W$, if $s_{i} w<w$,

$$
B_{w}^{-}(\lambda):=\left\{\tilde{e}_{i}^{k} b \mid k \geq 0, b \in B_{s_{i} w}^{-}(\lambda), \tilde{f}_{i} b=0\right\} \backslash\{0\} .
$$

Theorem $3.12([7])$. For $w \in W$, let $w=s_{i_{1}} \cdots s_{i_{n}}$ be an arbitrary reduced expression. Let $u_{\lambda}$ (resp. $\left.v_{\lambda^{\prime}}\right)$ be the highest (resp. lowest) weight vector of $B^{+}(\lambda)\left(\right.$ resp. $\left.B^{-}\left(\lambda^{\prime}\right)\right)$. Then

$$
\begin{aligned}
& B_{w}^{+}(\lambda)=\left\{\tilde{f}_{i_{1}}^{a(1)} \cdots \tilde{f}_{i_{n}}^{a(n)} u_{\lambda} \mid a(1), \ldots, a(n) \in \mathbb{Z}_{\geq 0}\right\} \backslash\{0\}, \\
& B_{w}^{-}\left(\lambda^{\prime}\right)=\left\{\tilde{e}_{i_{1}}^{a(1)} \cdots \tilde{e}_{i_{n}}^{a(n)} v_{\lambda^{\prime}} \mid a(1), \ldots, a(n) \in \mathbb{Z}_{\geq 0}\right\} \backslash\{0\} .
\end{aligned}
$$

Let $P^{+}$be the set of dominant weights. We set $P^{-}:=-P^{+}$.

Definition 3.13. Let $\mathcal{Y}(p)$ be the monomial realization of crystal associated with $p=\left(p_{i, j}\right)$. Suppose that $Y \in \mathcal{Y}(p)$ be a highest (resp. lowest) monomial with a weight $\lambda \in P^{ \pm}$. Thus, $Y$ is included in $\mu_{Y}\left(B_{w}^{ \pm}(\lambda)\right)$. Let us define the Demazure polynomial $D_{w}^{ \pm}[\lambda, Y ; C]$ associated with a monomial $Y, w \in W$ and coefficients $C=(c(b))_{b \in B_{w}^{ \pm}(\lambda)}\left(c(b) \in \mathbb{Z}_{>0}\right)$,

$$
D_{w}^{ \pm}[\lambda, Y ; C]:=\sum_{b \in B_{w}^{ \pm}(\lambda)} c(b) \mu_{Y}(b) .
$$

Remark 3.14. In this paper, we only treat the case that the coefficients $c(b)$ are equal to 1 for all $b \in B_{w}^{-}(\lambda)$ (see Theorem 5.6). But when $G \neq \mathrm{SL}_{r+1}(\mathbb{C})$ (for example, $G=\mathrm{Sp}_{2 r}(\mathbb{C})$ ), we need to treat the case $c(b)$ is not necessary equal to 1 for some $b \in B_{w}^{-}(\lambda)$. Therefore, we need non-trivial coefficients $c(b) \in \mathbb{Z}_{>0}$ in Definition 3.13.

\section{Cluster algebras and generalized minors}

In this section, we shall review the notions of cluster algebras. For all definitions in this section, see, e.g., $[1,4]$.

We set $[1, l]:=\{1,2, \ldots, l\}$ and $[-1,-l]:=\{-1,-2, \ldots,-l\}$ for $l \in \mathbb{Z}_{>0}$. For $n, m \in \mathbb{Z}_{>0}$, let $x_{1}, \ldots, x_{n}, x_{n+1}, \ldots, x_{n+m}$ be variables and $\mathcal{P}$ be a free multiplicative abelian group generated by $x_{n+1}, \ldots, x_{n+m}$. We set $\mathbb{Z} \mathcal{P}:=\mathbb{Z}\left[x_{n+1}^{ \pm 1}, \ldots, x_{n+m}^{ \pm 1}\right]$. Let $K:=\left\{\frac{g}{h} \mid g, h \in \mathbb{Z} \mathcal{P}, h \neq 0\right\}$ be the field of fractions of $\mathbb{Z} \mathcal{P}$, and $\mathcal{F}:=K\left(x_{1}, \ldots, x_{n}\right)$ be the field of rational functions.

\subsection{Cluster algebras of geometric type}

Definition 4.1. We set $n$-tuple of variables $\mathbf{x}=\left(x_{1}, \ldots, x_{n}\right)$. Let $\tilde{B}=\left(b_{i j}\right)_{1 \leq i \leq n, 1 \leq j \leq n+m}$ be $n \times(n+m)$ integer matrix whose principal part $B:=\left(b_{i j}\right)_{1 \leq i, j \leq n}$ is sign skew symmetric. Then a pair $\Sigma=(\mathbf{x}, \tilde{B})$ is called a seed, $\mathbf{x}$ a cluster and $x_{1}, \ldots, x_{n}$ cluster variables. For a seed $\Sigma=(\mathbf{x}, \tilde{B})$, principal part $B$ of $\tilde{B}$ is called the exchange matrix. 
Definition 4.2. For a seed $\Sigma=\left(\mathbf{x}, \tilde{B}=\left(b_{i j}\right)\right)$, an adjacent cluster in direction $k \in[1, n]$ is defined by

$$
\mathbf{x}_{k}=\left(\mathbf{x} \backslash\left\{x_{k}\right\}\right) \cup\left\{x_{k}^{\prime}\right\},
$$

where $x_{k}^{\prime}$ is the new cluster variable defined by the exchange relation

$$
x_{k} x_{k}^{\prime}=\prod_{1 \leq i \leq n+m, b_{k i}>0} x_{i}^{b_{k i}}+\prod_{1 \leq i \leq n+m, b_{k i}<0} x_{i}^{-b_{k i}} .
$$

Definition 4.3. Let $A=\left(a_{i j}\right), A^{\prime}=\left(a_{i j}^{\prime}\right)$ be two matrices of the same size. We say that $A^{\prime}$ is obtained from $A$ by the matrix mutation in direction $k$, and denote $A^{\prime}=\mu_{k}(A)$ if

$$
a_{i j}^{\prime}= \begin{cases}-a_{i j} & \text { if } i=k \text { or } j=k, \\ a_{i j}+\frac{\left|a_{i k}\right| a_{k j}+a_{i k}\left|a_{k j}\right|}{2} & \text { otherwise. }\end{cases}
$$

For $A, A^{\prime}$, if there exists a finite sequence $\left(k_{1}, \ldots, k_{s}\right), k_{i} \in[1, n]$, such that $A^{\prime}=\mu_{k_{1}} \cdots \mu_{k_{s}}(A)$, we say $A$ is mutation equivalent to $A^{\prime}$, and denote $A \cong A^{\prime}$.

Next proposition can be easily verified by the definition of $\mu_{k}$ :

Proposition 4.4 ([4, Proposition 3.6]). Let A be a skew symmetrizable matrix. Then any matrix that is mutation equivalent to $A$ is sign skew symmetric.

For a seed $\Sigma=(\mathbf{x}, \tilde{B})$, we say that the seed $\Sigma^{\prime}=\left(\mathbf{x}^{\prime}, \tilde{B}^{\prime}\right)$ is adjacent to $\Sigma$ if $\mathbf{x}^{\prime}$ is adjacent to $\mathbf{x}$ in direction $k$ and $\tilde{B}^{\prime}=\mu_{k}(\tilde{B})$. Two seeds $\Sigma$ and $\Sigma_{0}$ are mutation equivalent if one of them can be obtained from another seed by a sequence of pairwise adjacent seeds and we denote $\Sigma \sim \Sigma_{0}$.

Now let us define a cluster algebra of geometric type.

Definition 4.5. Let $\tilde{B}$ be a skew symmetrizable matrix, and $\Sigma=(\mathbf{x}, \tilde{B})$ a seed. We set $\mathbb{A}:=\mathbb{Z}\left[x_{n+1}, \ldots, x_{n+m}\right]$. The cluster algebra (of geometric type) $\mathcal{A}=\mathcal{A}(\Sigma)$ over $\mathbb{A}$ associated with seed $\Sigma$ is defined as the $\mathbb{A}$-subalgebra of $\mathcal{F}$ generated by all cluster variables in all seeds which are mutation equivalent to $\Sigma$.

For a seed $\Sigma$, we define $\mathbb{Z} \mathcal{P}$-subalgebra $\mathcal{U}(\Sigma)$ of $\mathcal{F}$ by

$$
\mathcal{U}(\Sigma):=\mathbb{Z} \mathcal{P}\left[\mathbf{x}^{ \pm 1}\right] \cap \mathbb{Z} \mathcal{P}\left[\mathbf{x}_{1}^{ \pm 1}\right] \cap \cdots \cap \mathbb{Z} \mathcal{P}\left[\mathbf{x}_{n}^{ \pm 1}\right]
$$

Here, $\mathbb{Z} \mathcal{P}\left[\mathbf{x}^{ \pm 1}\right]$ is the Laurent polynomial ring in $\mathbf{x}$.

Definition 4.6. Let $\Sigma_{0}=(\mathbf{x}, \tilde{B})$ be a seed such that $\tilde{B}$ is skew symmetrizable. We define an upper cluster algebra $\overline{\mathcal{A}}=\overline{\mathcal{A}}\left(\Sigma_{0}\right)$ as the intersection of the subalgebras $\mathcal{U}(\Sigma)$ for all seeds $\Sigma \sim \Sigma_{0}$.

Following the inclusion relation holds [1]:

$$
\mathcal{A}(\Sigma) \subset \overline{\mathcal{A}}(\Sigma)
$$

\subsection{Cluster algebras on double Bruhat cells of type A}

As in Section 2, let $G=\mathrm{SL}_{r+1}(\mathbb{C})$ be the simple algebraic group of type $\mathrm{A}_{r}$ and $W=\mathfrak{S}_{r+1}$ be its Weyl group. We set $u \in W$ and its reduced word $\mathbf{i}$ as in (2.1) and (2.2):

$$
u=\underbrace{s_{1} s_{2} \cdots s_{r}}_{1^{\text {st }} \text { cycle }} \underbrace{s_{1} \cdots s_{r-1}}_{2^{\text {nd }} \text { cycle }} \cdots \underbrace{s_{1} \cdots s_{r-m+2}}_{(m-1)^{\text {th }} \text { cycle }} \underbrace{s_{1} \cdots s_{i_{n}}}_{m^{\text {th }} \text { cycle }},
$$




$$
\mathbf{i}=(\underbrace{1, \ldots, r}_{1^{\text {st }} \text { cycle }}, \underbrace{1, \ldots,(r-1)}_{2^{\text {nd }} \text { cycle }}, \ldots, \underbrace{1, \ldots,(r-m+2)}_{(m-1)^{\text {th }} \text { cycle }}, \underbrace{1, \ldots, i_{n}}_{m^{\text {th }} \text { cycle }}) .
$$

We shall constitute the upper cluster algebra $\overline{\mathcal{A}}(\mathbf{i})$ from i. Let $i_{k}, k \in[1, l(u)]$, be the $k^{\text {th }}$ index of $\mathbf{i}$ from the left.

At first, we define a set $e(\mathbf{i})$ as

$$
e(\mathbf{i}):=[-1,-r] \cup\left\{k \mid \text { there exist some } l>k \text { such that } i_{k}=i_{l}\right\} .
$$

Next, let us define a matrix $\tilde{B}=\tilde{B}(\mathbf{i})$.

Definition 4.7. Let $\tilde{B}(\mathbf{i})$ be an integer matrix with rows labeled by all the indices in $[-1,-r] \cup$ $[1, l(u)]$ and columns labeled by all the indices in $e(\mathbf{i})$. For $k \in[-1,-r] \cup[1, l(u)]$ and $l \in e(\mathbf{i})$, an entry $b_{k l}$ of $\tilde{B}(\mathbf{i})$ is determined as follows

$$
b_{k l}= \begin{cases}-\operatorname{sgn}\left((k-l) \cdot i_{p}\right) & \text { if } p=q, \\ -\operatorname{sgn}\left((k-l) \cdot i_{p} \cdot a_{\left|i_{k}\right|\left|i_{l}\right|}\right) & \text { if } p<q \quad \text { and } \operatorname{sgn}\left(i_{p} \cdot i_{q}\right)(k-l)\left(k^{+}-l^{+}\right)>0, \\ 0 & \text { otherwise. }\end{cases}
$$

Proposition 4.8 ([1, Proposition 2.6]). The matrix $\tilde{B}(\mathbf{i})$ is skew symmetrizable.

By Proposition 4.4, Definition 4.6 and Proposition 4.8, we can construct the upper cluster algebra from $\tilde{B}(\mathbf{i})$ :

Definition 4.9. We denote this upper cluster algebra by $\bar{A}(\mathbf{i})$.

Now, we set $\overline{\mathcal{A}}(\mathbf{i})_{\mathbb{C}}:=\overline{\mathcal{A}}(\mathbf{i}) \otimes \mathbb{C}$ and $\mathcal{F}_{\mathbb{C}}:=\mathcal{F} \otimes \mathbb{C}$. It is known that the coordinate ring $\mathbb{C}\left[G^{u, e}\right]$ of the double Bruhat cell is isomorphic to $\overline{\mathcal{A}}(\mathbf{i})_{\mathbb{C}}$ (Theorem 4.11). To describe this isomorphism explicitly, we need generalized minors. For $k \in[1, l(u)]$, let $i_{k}$ be the $k^{\text {th }}$ index of $\mathbf{i}(4.2)$ from the left, and we suppose that it belongs to the $m^{\text {th }}$ cycle. We set

$$
u_{\leq k}=u_{\leq k}(\mathbf{i}):=\underbrace{s_{1} s_{2} \cdots s_{r}}_{1^{\text {st }} \text { cycle }} \underbrace{s_{1} \cdots s_{r-1}}_{2^{\text {nd }} \text { cycle }} \cdots \underbrace{s_{1} \cdots s_{i_{k}}}_{m^{\text {th }} \text { cycle }} .
$$

For $k \in[-1,-r]$, we set $u_{\leq k}:=e$ and $i_{k}:=k$. In the case $G=\mathrm{SL}_{r+1}(\mathbb{C})$, the generalized minors are nothing but the ordinary minors of a matrix:

Definition 4.10 ([1]). For $x \in G=\mathrm{SL}_{r+1}(\mathbb{C})$ and $k \in[-1,-r] \cup[1, l(u)]$, we define the generalized minor $\Delta(k ; \mathbf{i})(x)$ as the minor of $x$ whose rows (resp. columns) are labeled by the elements of the set $u_{\leq k}\left(\left[1,\left|i_{k}\right|\right]\right)$ (resp. $\left.\left[1,\left|i_{k}\right|\right]\right)$.

Finally, we set

$$
F(\mathbf{i}):=\{\Delta(k ; \mathbf{i}) \mid k \in[-1,-r] \cup[1, l(u)]\} .
$$

It is known that the set $F(\mathbf{i})$ is an algebraically independent generating set for the field of rational functions $\mathbb{C}\left(G^{u, e}\right)$ [3, Theorem 1.12]. Then, we have the following theorem.

Theorem 4.11 ([1, Theorem 2.10]). The isomorphism of fields $\varphi: F_{\mathbb{C}} \rightarrow \mathbb{C}\left(G^{u, e}\right)$ defined by $\varphi\left(x_{k}\right)=\Delta(k ; \mathbf{i}), k \in[-1,-r] \cup[1, l(u)]$, restricts to an isomorphism of algebras $\overline{\mathcal{A}}(\mathbf{i})_{\mathbb{C}} \rightarrow \mathbb{C}\left[G^{u, e}\right]$. 


\section{$5 \quad$ Generalized minors and crystals}

In the rest of the paper, we consider the case $G=\mathrm{SL}_{r+1}(\mathbb{C})$, and let $u \in W$ and its reduced word $\mathbf{i}$ as in (4.1) and (4.2):

$$
\begin{aligned}
& u=s_{1} s_{2} \cdots s_{r} s_{1} \cdots s_{r-1} \cdots s_{1} \cdots s_{r-m+2} s_{1} \cdots s_{i_{n}}, \\
& \mathbf{i}=(\underbrace{1, \ldots, r}_{1^{\text {st }} \text { cycle }}, \underbrace{1, \ldots,(r-1)}_{2^{\text {nd }} \text { cycle }}, \ldots, \underbrace{1, \ldots,(r-m+2)}_{(m-1)^{\text {th }} \text { cycle }}, \underbrace{1, \ldots, i_{n}}_{m^{\text {th }} \text { cycle }}),
\end{aligned}
$$

that is, $\mathbf{i}$ is the left factor of $(1,2,3, \ldots, r, 1,2,3, \ldots,(r-1), \ldots, 1,2,1)$. Let $i_{k}$ be the $k^{\text {th }}$ index of $\mathbf{i}$ from the left, and belong to $m^{\text {th }}$ cycle. As we shall show in Lemma 5.4, we may assume $i_{n}=i_{k}$.

By Theorem 4.11, we can regard $\mathbb{C}\left[G^{u, e}\right]$ as an upper cluster algebra and $\{\Delta(k ; \mathbf{i})\}$ as its cluster variables belonging to a given initial seed. Each $\Delta(k ; \mathbf{i})$ is a regular function on $G^{u, e}$. On the other hand, by Proposition 2.4 (resp. Theorem 2.3), we can consider $\Delta(k ; \mathbf{i})$ as a function on $H \times\left(\mathbb{C}^{\times}\right)^{l(u)}\left(\right.$ resp. $\left.\left(\mathbb{C}^{\times}\right)^{l(u)}\right)$. Then we change the variables of $\{\Delta(k ; \mathbf{i})\}$ as follows:

Definition 5.1. For $a \in H$ and $\mathbf{t}, \tau \in\left(\mathbb{C}^{\times}\right)^{l(u)}$ we set

$$
\begin{aligned}
& \Delta^{G}(k ; \mathbf{i})(a, \mathbf{t}):=\left(\Delta(k ; \mathbf{i}) \circ \bar{x}_{\mathbf{i}}^{G}\right)(a, \mathbf{t}), \\
& \Delta^{L}(k ; \mathbf{i})(\tau):=\left(\Delta(k ; \mathbf{i}) \circ x_{\mathbf{i}}^{L}\right)(\tau),
\end{aligned}
$$

where $\mathbf{t}=\left(t_{1}, \ldots, t_{l(u)}\right), \tau=\left(\tau_{1}, \ldots, \tau_{l(u)}\right)$.

We will describe the function $\Delta^{L}(k ; \mathbf{i})(\tau)$ by using monomial realizations of Demazure crystals.

\subsection{Generalized minor $\Delta^{G}(k ; \mathrm{i})(a, \mathrm{t})$}

In this subsection, we shall prove that $\Delta^{G}(k ; \mathbf{i})(a, \mathbf{t})$ is immediately obtained from $\Delta^{L}(k ; \mathbf{i})$ :

Proposition 5.2. We set $d:=i_{k}$. For $a=\operatorname{diag}\left(a_{1}, \ldots, a_{r+1}\right) \in H$,

$$
\Delta^{G}(k ; \mathbf{i})(a, \mathbf{t})=a_{m^{\prime}+1} \cdots a_{m^{\prime}+d} \Delta^{L}(k ; \mathbf{i})(\mathbf{t}) .
$$

This proposition follows from the following lemma:

Lemma 5.3. In the above setting, $\Delta^{G}(k ; \mathbf{i})(a, \mathbf{t})\left(\right.$ resp. $\left.\Delta^{L}(k ; \mathbf{i})(\tau)\right)$ is given as a minor whose row are labeled by the set $\left\{m^{\prime}+1, \ldots, m^{\prime}+d\right\}$ and column are labeled by the set $\{1, \ldots, d\}$ of the matrix

$$
\begin{aligned}
& a \underbrace{x_{-1}\left(t_{1}\right) x_{-2}\left(t_{2}\right) \cdots x_{-r}\left(t_{r}\right)}_{1^{\text {st }} \text { cycle }} \underbrace{x_{-1}\left(t_{l_{1}+1}\right) \cdots x_{-(r-1)}\left(t_{l_{1}+r-1}\right)}_{m^{\text {th }} \text { cycle }} \cdots \\
& \times \underbrace{x_{-1}\left(t_{l_{m-1}+1}\right) \cdots x_{-i_{n}}\left(t_{l_{m-1}+i_{n}}\right)},
\end{aligned}
$$

resp.

$$
\begin{aligned}
& \underbrace{x_{-1}\left(\tau_{1}\right) x_{-2}\left(\tau_{2}\right) \cdots x_{-r}\left(\tau_{r}\right)}_{1^{\text {st }} \text { cycle }} \underbrace{x_{-1}\left(\tau_{l_{1}+1}\right) \cdots x_{-(r-1)}\left(\tau_{l_{1}+r-1}\right)}_{2^{\text {nd }} \text { cycle }} \cdots \\
& \times \underbrace{x_{-1}\left(\tau_{l_{m-1}+1}\right) \cdots x_{-i_{n}}\left(\tau_{l_{m-1}+i_{n}}\right)}_{\text {cycle }}
\end{aligned}
$$


Proof. Let us prove this lemma for $\Delta^{L}(k ; \mathbf{i})(\tau)$ since the case for $\Delta^{G}(k ; \mathbf{i})(a, \mathbf{t})$ is proven similarly. By the definition (4.3) of $u_{\leq k}$ and $i_{k}=d$, we have

$$
\begin{aligned}
u_{\leq k}\left[1,\left|i_{k}\right|\right] & =u_{\leq k}\{1, \ldots, d\}=\underbrace{s_{1} \cdots s_{r}}_{1^{\text {st }} \text { cycle }} \cdots \underbrace{s_{1} \cdots s_{r-m^{\prime}+2}}_{\left(m^{\prime}-1\right)^{\text {th }} \text { cycle } m^{\prime \text { th }} \text { cycle }} \underbrace{s_{1} \cdots s_{d}}_{1}\{1, \ldots, d\} \\
& =\left\{m^{\prime}+1, \ldots, m^{\prime}+d\right\} .
\end{aligned}
$$

Hence, by Theorem 2.3 and Definition $4.10, \Delta^{L}(k ; \mathbf{i})(\tau)$ is given as a minor whose row (resp. column) are labeled by the set $\left\{m^{\prime}+1, \ldots, m^{\prime}+d\right\}$ (resp. $\left.\{1, \ldots, d\}\right)$ of the matrix

$$
\begin{aligned}
& \underbrace{x_{-1}\left(\tau_{1}\right) x_{-2}\left(\tau_{2}\right) \cdots x_{-r}\left(\tau_{r}\right)}_{1^{\text {st }} \text { cycle }} \underbrace{x_{-1}^{\text {nd }}\left(\tau_{l_{1}+1}\right) \cdots x_{-(r-1)}\left(\tau_{l_{1}+r-1}\right)}_{2^{\text {th }} \text { cycle }} \cdots \\
& \times \underbrace{x_{-1}\left(\tau_{l_{m-1}+1}\right) \cdots x_{-i_{n}}\left(\tau_{l_{m-1}+i_{n}}\right)},
\end{aligned}
$$

which implies the desired result.

In the rest of the paper, we will treat $\Delta^{L}(k ; \mathbf{i})(\tau)$ only by Proposition 5.2 .

\subsection{Generalized minor $\Delta^{L}(k ; \mathrm{i})(\tau)$}

Lemma 5.4. Let $u$ and $\mathbf{i}=\left(1, \ldots, i_{n}\right)$ be as in the form (5.1) and (5.2) respectively. For $i_{n+1} \in$ $[1, r]$ and $u^{\prime}:=u s_{i_{n+1}} \in W\left(l\left(u^{\prime}\right)>l(u)\right)$ we set the reduced word for $u^{\prime}$ as $\mathbf{i}^{\prime}:=\left(1, \ldots, i_{n}, i_{n+1}\right)$. We also set $\tau=\left(\tau_{1}, \ldots, \tau_{n}\right)$ and $\tau^{\prime}=\left(\tau_{1}, \ldots, \tau_{n}, \tau_{n+1}\right)$. For an integer $k, 1 \leq k \leq n$, if $d:=i_{k} \neq i_{n+1}$, then $\Delta^{L}\left(k ; \mathbf{i}^{\prime}\right)\left(\tau^{\prime}\right)$ does not depend on $\tau_{n+1}$, so we can regard it as a function on $H \times\left(\mathbb{C}^{\times}\right)^{n}$. Furthermore, we have

$$
\Delta^{L}(k ; \mathbf{i})(\tau)=\Delta^{L}\left(k ; \mathbf{i}^{\prime}\right)\left(\tau^{\prime}\right) .
$$

Proof. We denote the matrix (5.3) by $T=\left(T_{i, j}\right)_{i, j \in[1, r+1]}$ :

$$
T=\left(T_{i, j}\right)_{i, j \in[1, r+1]}:=x_{-1}\left(\tau_{1}\right) x_{-2}\left(\tau_{2}\right) \cdots x_{-i_{n}}\left(\tau_{n}\right) .
$$

We also define the submatrix $T_{u}$ of the matrix $T$ whose rows (resp. columns) are labeled by the set $\left\{m^{\prime}+1, \ldots, m^{\prime}+d\right\}$ (resp. $\left.\{1, \ldots, d\}\right)$, that is

$$
T_{u}:=\left(\begin{array}{ccc}
T_{m^{\prime}+1,1} & \cdots & T_{m^{\prime}+1, d} \\
\vdots & \vdots & \vdots \\
T_{m^{\prime}+d, 1} & \cdots & T_{m^{\prime}+d, d}
\end{array}\right) .
$$

By Lemma $5.3, \Delta^{L}(k ; \mathbf{i})(\tau)$ is the determinant of $T_{u}$.

Similarly, we define the submatrix $T_{u^{\prime}}^{\prime}$ of the matrix $T^{\prime}:=T \cdot x_{-i_{n+1}}\left(\tau_{n+1}\right)$ whose rows (resp. columns) are labeled by the set $\left\{m^{\prime}+1, \ldots, m^{\prime}+d\right\}$ (resp. $\{1, \ldots, d\}$ ). By Lemma 5.3, $\Delta^{L}\left(k ; \mathbf{i}^{\prime}\right)\left(\tau^{\prime}\right)$ is the determinant of $T_{u^{\prime}}^{\prime}$.

Using the explicit form of $x_{-i}(t)$ in (2.4), we have the following relation: If $i_{n+1}<i_{k}=d$, then

$$
T_{u^{\prime}}^{\prime}=\left(\begin{array}{cccccc}
T_{m^{\prime}+1,1} & \ldots & \tau_{n+1}^{-1} T_{m^{\prime}+1, i_{n+1}}+T_{m^{\prime}+1, i_{n+1}+1} & \tau_{n+1} T_{m^{\prime}+1, i_{n+1}+1} & \ldots & T_{m^{\prime}+1, d} \\
\vdots & \vdots & \vdots & \vdots & \vdots & \vdots \\
T_{m^{\prime}+d, 1} & \ldots & \tau_{n+1}^{-1} T_{m^{\prime}+1, i_{n+1}}+T_{m^{\prime}+1, i_{n+1}+1} & \tau_{n+1} T_{m^{\prime}+1, i_{n+1}+1} & \ldots & T_{m^{\prime}+d, d}
\end{array}\right) .
$$

If $i_{n+1}>i_{k}=d$, then $T_{u^{\prime}}^{\prime}=T_{u}$. Therefore, it is clear that for both cases, $\operatorname{det} T_{u^{\prime}}^{\prime}=\operatorname{det} T_{u}$, which means $\Delta^{L}\left(k ; \mathbf{i}^{\prime}\right)\left(\tau^{\prime}\right)=\Delta^{L}(k ; \mathbf{i})(\tau)$. 
By this lemma, when we calculate $\Delta^{L}(k ; \mathbf{i})(\tau)$, we may assume that $i_{n}=i_{k}$ without loss of generality.

We set $l_{i}=\sum_{k=1}^{i}(r-k+1)(1 \leq i \leq m-1)$, and change the variables $\left\{Y_{m, j}\right\}$ to $\left\{\tau_{l_{m}+j}\right\}$ as in Section 3.1.

Remark 5.5. For

$$
\mathbf{i}=(\underbrace{1, \ldots, r}_{1^{\text {st }} \text { cycle }}, \underbrace{1, \ldots,(r-1)}_{2^{\text {nd }} \text { cycle }}, \ldots, \underbrace{1, \ldots,(r-m+2)}_{(m-1)^{\text {th }} \text { cycle }}, \underbrace{1, \ldots, i_{n}}_{m^{\text {th }} \text { cycle }})
$$

$\left(l_{i}+j\right)^{\text {th }}$ index of $\mathbf{i}$ from the left is $j$ which belongs to $(i+1)^{\text {th }}$ cycle $(1 \leq j \leq r-i-1)$.

The following theorem is our main result. We describe $\Delta^{L}(k ; \mathbf{i})(\tau)$ as a Demazure polynomial $D_{w}^{-}$in Definition 3.13.

Theorem 5.6. In the above setting, we set $d:=i_{k}=i_{n}$ and suppose that $i_{k}$ belongs to $m^{\text {th }}$ cycle in $\mathbf{i}$ (5.2). We also set

$$
Y:=\frac{1}{\tau_{l_{m-1}+d} \tau_{l_{m-2}+d} \cdots \tau_{l_{m^{\prime}}+d}} .
$$

Then we have

$$
\Delta^{L}(k ; \mathbf{i})(\tau)=\sum_{x \in B_{u_{\leq k}}^{-}\left(\left(m^{\prime}-m\right) \Lambda_{d}\right)} \mu_{Y}(x)=D_{u_{\leq k}}^{-}\left[\left(m^{\prime}-m\right) \Lambda_{d}, Y ; \mathbf{1}\right],
$$

where $\mu_{Y}(x)$ is an embedding of $x \in B_{u_{\leq k}}^{-}\left(\left(m^{\prime}-m\right) \Lambda_{d}\right)$ as in Example 3.5, and 1 means all $c(b) \equiv 1$ (Definition 3.13).

We shall prove this theorem in Section 6. In particular, we can explicitly write down $\Delta^{L}(k ; \mathbf{i})(\tau)$ in the case $i_{k}=1$.

Theorem 5.7. If $i_{k}=1$ and $i_{k}$ is in the $m^{\text {th }}$ cycle in $\mathbf{i}(5.2)$, then we have

$$
\begin{aligned}
\Delta^{L}(k ; \mathbf{i})(\tau) & =\sum_{0 \leq j_{1}<\cdots<j_{m^{\prime}} \leq m-1} \prod_{i=0}^{j_{1}-1} \frac{1}{\tau_{l_{m-1-i}+1}} \prod_{i=j_{1}+1}^{j_{2}-1} \frac{\tau_{l_{m-1-i}+1}}{\tau_{l_{m-1-i}+2}} \cdots \prod_{i=j_{m^{\prime}+1}}^{m-1} \frac{\tau_{l_{m-1-i}+m^{\prime}}}{\tau_{l_{m-1-i}+m^{\prime}+1}} \\
& =D_{u_{\leq k}}^{-}\left[\left(m^{\prime}-m\right) \Lambda_{1}, \frac{1}{\tau_{l_{m-1}+1} \tau_{l_{m-2}+1} \cdots \tau_{l_{m^{\prime}+1}+1} \tau_{l_{m^{\prime}}+1}} ; \mathbf{1}\right] .
\end{aligned}
$$

We will prove this theorem in Section 6.5.

\section{The proof of Theorems 5.6 and 5.7}

In this section, we shall give the proof of Theorems 5.6 and 5.7. In Sections 6.1-6.4, we will prove Theorem 5.6. In Section 6.5, we prove Theorem 5.7. We use the same notation as in Section 5.2: $l_{i}=\sum_{k=1}^{i}(r-k+1), 1 \leq i \leq m-1$. 


\subsection{The set $X_{d}\left(m, m^{\prime}\right)$ of paths}

In this subsection, we shall introduce a set $X_{d}\left(m, m^{\prime}\right)$ of "paths" which correspond to the terms of $\Delta^{L}(k ; \mathbf{i})(\tau)$. Let $m, m^{\prime}$ and $d$ be the positive integers as in Section 5.2.

Definition 6.1. Let us define the directed graph $\left(V_{d}, E_{d}\right)$ as follows. We define the set $V_{d}=$ $V_{d}\left(m, m^{\prime}\right)$ of vertices as

$$
\begin{aligned}
V_{d}\left(m, m^{\prime}\right):=\{ & \left(m-s ; a_{1}^{(s)}, a_{2}^{(s)}, \ldots, a_{d}^{(s)}\right) \mid \\
& \left.0 \leq s \leq m, a_{i}^{(s)} \in \mathbb{Z}, 1 \leq a_{1}^{(s)}<a_{2}^{(s)}<\cdots<a_{d}^{(s)} \leq d+s\right\} .
\end{aligned}
$$

Note that $a_{1}^{(0)}=1, a_{2}^{(0)}=2, \ldots, a_{d}^{(0)}=d$ by $1 \leq a_{1}^{(0)}<a_{2}^{(0)}<\cdots<a_{d}^{(0)} \leq d$. And we define the set $E_{d}=E_{d}\left(m, m^{\prime}\right)$ of directed edges as

$$
\begin{aligned}
E_{d}\left(m, m^{\prime}\right):=\{ & \left(m-s ; a_{1}^{(s)}, \ldots, a_{d}^{(s)}\right) \rightarrow\left(m-s-1 ; a_{1}^{(s+1)}, \ldots, a_{d}^{(s+1)}\right) \mid \\
& \left.1 \leq s \leq m, 1 \leq i \leq d, a_{i}^{(s+1)}=a_{i}^{(s)} \text { or } a_{i}^{(s)}+1\right\} .
\end{aligned}
$$

Definition 6.2. Let $X_{d}\left(m, m^{\prime}\right)$ be the set of directed paths from $(m ; 1, \ldots, d)$ to $\left(0 ; m^{\prime}+1\right.$, $\left.m^{\prime}+2, \ldots, m^{\prime}+d\right)$ in $\left(V_{d}, E_{d}\right)$. In other word, any path $p \in X_{d}\left(m, m^{\prime}\right)$

$$
\begin{aligned}
p= & \left(m ; a_{1}^{(0)}, \ldots, a_{d}^{(0)}\right) \rightarrow\left(m-1 ; a_{1}^{(1)}, \ldots, a_{d}^{(1)}\right) \rightarrow\left(m-2 ; a_{1}^{(2)}, \ldots, a_{d}^{(2)}\right) \rightarrow \cdots \\
& \rightarrow\left(1 ; a_{1}^{(m-1)}, \ldots, a_{d}^{(m-1)}\right) \rightarrow\left(0 ; a_{1}^{(m)}, \ldots, a_{d}^{(m)}\right)
\end{aligned}
$$

are characterized by the following conditions

(i) $a_{i}^{(s)} \in \mathbb{Z}_{\geq 1}$,

(ii) $a_{1}^{(s)}<a_{2}^{(s)}<\cdots<a_{d}^{(s)}$,

(iii) $a_{i}^{(s+1)}=a_{i}^{(s)}$ or $a_{i}^{(s)}+1$,

(iv) $a_{i}^{(0)}=i, a_{i}^{(m)}=m^{\prime}+i$.

Remark 6.3. By Definition 6.2(ii), (iii) and (iv), we have

$$
a_{i}^{(s)} \leq a_{d}^{(s)} \leq a_{d}^{(m)}=m^{\prime}+d
$$

for any $1 \leq i \leq d$ and $0 \leq s \leq m$.

Let us define a Laurent monomial associated with each path in $X_{d}\left(m, m^{\prime}\right)$.

Definition 6.4. Let $p \in X_{d}\left(m, m^{\prime}\right)$ be a path

$$
\begin{aligned}
p= & \left(m ; a_{1}^{(0)}, \ldots, a_{d}^{(0)}\right) \rightarrow\left(m-1 ; a_{1}^{(1)}, \ldots, a_{d}^{(1)}\right) \rightarrow\left(m-2 ; a_{1}^{(2)}, \ldots, a_{d}^{(2)}\right) \rightarrow \cdots \\
& \rightarrow\left(1 ; a_{1}^{(m-1)}, \ldots, a_{d}^{(m-1)}\right) \rightarrow\left(0 ; a_{1}^{(m)}, \ldots, a_{d}^{(m)}\right) .
\end{aligned}
$$

(i) For each $0 \leq s \leq m$, we define the label of the edge $\left(m-s ; a_{1}^{(s)}, a_{2}^{(s)}, \ldots, a_{d}^{(s)}\right) \rightarrow(m-s-1$; $\left.a_{1}^{(s+1)}, a_{2}^{(s+1)}, \ldots, a_{d}^{(s+1)}\right)$ as the Laurent monomial

$$
\prod_{i=1}^{d} \frac{\tau_{l_{m-s-1}+a_{i}^{(s+1)}-1}}{\tau_{l_{m-s-1}+a_{i}^{(s)}}}
$$


(ii) And we define the label $Q(p)$ of the path $p$ as the product of them

$$
Q(p):=\prod_{s=0}^{m-1}\left(\prod_{i=1}^{d} \frac{\tau_{l_{m-s-1}+a_{i}^{(s+1)}-1}}{\tau_{l_{m-s-1}+a_{i}^{(s)}}}\right) .
$$

Example 6.5. Let $m=3, m^{\prime}=2, d=2$. We can describe $X_{2}(3,2)$ and its labels as follows

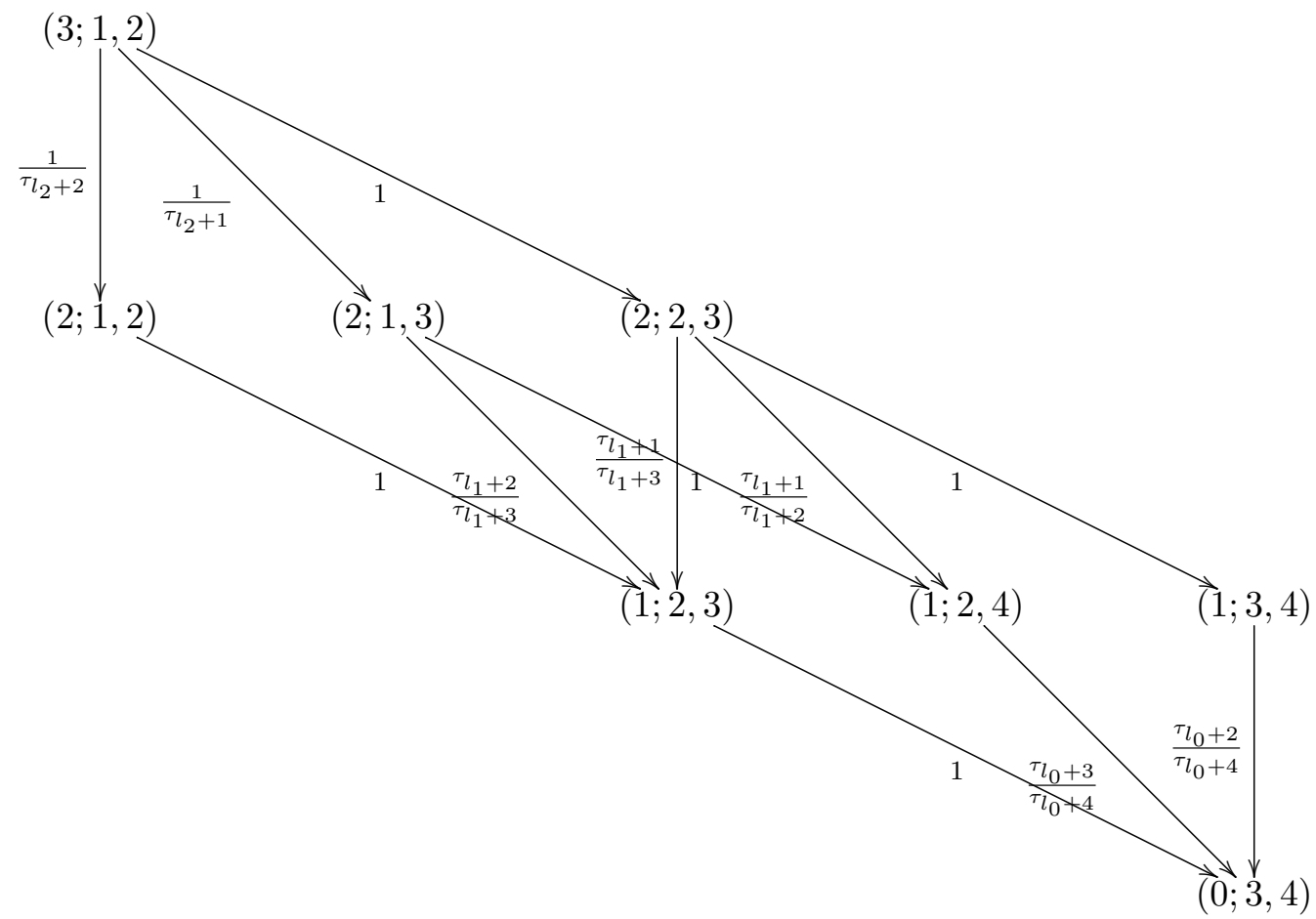

Each edge has the label written on the left side of it. The paths in $X_{2}(3,2)$ are as follows

$$
\begin{aligned}
& p_{1}=(3 ; 1,2) \rightarrow(2 ; 1,2) \rightarrow(1 ; 2,3) \rightarrow(0 ; 3,4), \\
& p_{2}=(3 ; 1,2) \rightarrow(2 ; 1,3) \rightarrow(1 ; 2,3) \rightarrow(0 ; 3,4), \\
& p_{3}=(3 ; 1,2) \rightarrow(2 ; 1,3) \rightarrow(1 ; 2,4) \rightarrow(0 ; 3,4), \\
& p_{4}=(3 ; 1,2) \rightarrow(2 ; 2,3) \rightarrow(1 ; 2,3) \rightarrow(0 ; 3,4), \\
& p_{5}=(3 ; 1,2) \rightarrow(2 ; 2,3) \rightarrow(1 ; 2,4) \rightarrow(0 ; 3,4), \\
& p_{6}=(3 ; 1,2) \rightarrow(2 ; 2,3) \rightarrow(1 ; 3,4) \rightarrow(0 ; 3,4) .
\end{aligned}
$$

We have

$$
\begin{aligned}
& Q\left(p_{1}\right)=\frac{1}{\tau_{l_{2}+2}}, \quad Q\left(p_{2}\right)=\frac{\tau_{l_{1}+2}}{\tau_{l_{2}+1} \tau_{l_{1}+3}}, \quad Q\left(p_{3}\right)=\frac{\tau_{l_{0}+3}}{\tau_{l_{2}+1} \tau_{l_{0}+4}}, \\
& Q\left(p_{4}\right)=\frac{\tau_{l_{1}+1}}{\tau_{l_{1}+3}}, \quad Q\left(p_{5}\right)=\frac{\tau_{l_{1}+1} \tau_{l_{0}+3}}{\tau_{l_{1}+2} \tau_{l_{0}+4}}, \quad Q\left(p_{6}\right)=\frac{\tau_{l_{0}+2}}{\tau_{l_{0}+4}} .
\end{aligned}
$$

Definition 6.6. For each path $p \in X_{d}\left(m, m^{\prime}\right)$

$$
\begin{aligned}
p= & \left(m ; a_{1}^{(0)}, \ldots, a_{d}^{(0)}\right) \rightarrow\left(m-1 ; a_{1}^{(1)}, \ldots, a_{d}^{(1)}\right) \rightarrow\left(m-2 ; a_{1}^{(2)}, \ldots, a_{d}^{(2)}\right) \rightarrow \cdots \\
& \rightarrow\left(1 ; a_{1}^{(m-1)}, \ldots, a_{d}^{(m-1)}\right) \rightarrow\left(0 ; a_{1}^{(m)}, \ldots, a_{d}^{(m)}\right)
\end{aligned}
$$

and $i \in\{1, \ldots, d\}$, we call the following sequence

$$
a_{i}^{(0)} \rightarrow a_{i}^{(1)} \rightarrow a_{i}^{(2)} \rightarrow \cdots \rightarrow a_{i}^{(m)}
$$

an $i$-sequence of $p$. 
Example 6.7. In the setting of Example 6.5, $p_{1}:=(3 ; 1,2) \rightarrow(2 ; 1,2) \rightarrow(1 ; 2,3) \rightarrow(0 ; 3,4)$. Then, 1-sequence of $p_{1}$ is

$$
1 \rightarrow 1 \rightarrow 2 \rightarrow 3
$$

and 2 -sequence of $p_{1}$ is

$$
2 \rightarrow 2 \rightarrow 3 \rightarrow 4
$$

\subsection{One-to-one correspondence between paths in $X_{d}\left(m, m^{\prime}\right)$ and terms of $\Delta^{L}(k ; i)(\tau)$}

Proposition 6.8. We use the setting (5.1), (5.2) and the notations in Section 5. Then, we have the following

$$
\Delta^{L}(k ; \mathbf{i})(\tau)=\sum_{p \in X_{d}\left(m, m^{\prime}\right)} Q(p) .
$$

To prove this proposition, we need the following preparations. For $s(0 \leq s \leq m-1)$, we define a matrix $x^{(s)}(\tau)$ as

$$
\begin{aligned}
x^{(s)}(\tau):= & \underbrace{x_{-1}\left(\tau_{1}\right) x_{-2}\left(\tau_{2}\right) \cdots x_{-r}\left(\tau_{r}\right)}_{1^{\text {st }} \text { cycle }} \underbrace{x_{-1}\left(\tau_{l_{1}+1}\right) \cdots x_{-(r-1)}\left(\tau_{l_{1}+r-1}\right)}_{2^{\text {nd }}} \cdots \\
& \times \underbrace{x_{-1}\left(\tau_{l_{s-1}+1}\right) \cdots x_{-(r-s+1)}\left(\tau_{l_{s-1}+r-s+1}\right)}_{s^{\text {th }} \text { cycle }},
\end{aligned}
$$

where we understand $x^{(0)}(\tau)$ means identity matrix. We also define

$$
\begin{aligned}
x^{(m)}(\tau):= & \underbrace{x_{-1}\left(\tau_{1}\right) x_{-2}\left(\tau_{2}\right) \cdots x_{-r}\left(\tau_{r}\right)}_{1^{\text {st }} \text { cycle }} \underbrace{x_{-1}^{\text {nd }}\left(\tau_{l_{1}+1}\right) \cdots x_{-(r-1)}\left(\tau_{l_{1}+r-1}\right)}_{m^{\text {th }} \text { cycle }} \cdots \\
& \times \underbrace{x_{-1}\left(\tau_{l_{m-1}+1}\right) \cdots x_{-i_{n}}\left(\tau_{l_{m-1}+i_{n}}\right)}_{\text {cycle }},
\end{aligned}
$$

which is equal to the matrix in (5.3).

For $x^{(s)}(\tau)=\left(x_{i, j}^{(s)}\right)_{i, j \in[1, r+1]}$, we define the $d$ dimensional column vector $D(s ; p)$

$$
D(s ; p):=\left(\begin{array}{c}
x_{m^{\prime}+1, p}^{(s)} \\
\vdots \\
x_{m^{\prime}+d, p}^{(s)}
\end{array}\right) \in \mathbb{C}^{d} .
$$

Note that, by the explicit form of $x_{-i}(\tau)$ in (2.4), multiplying $x_{-i}(\tau)$ from the right gives an elementary transformation of a matrix. Therefore, we get

$$
D(s ; p)= \begin{cases}\frac{1}{\tau_{l_{s-1}+p}} D(s-1 ; p)+D(s-1 ; p+1) & \text { if } \quad p=1, \\ \frac{\tau_{l_{s-1}+p-1}}{\tau_{l_{s-1}+p}} D(s-1 ; p)+D(s-1 ; p+1) & \text { if } \quad p>1,\end{cases}
$$

for $1 \leq s \leq m$. For $0 \leq s \leq m$ and $1 \leq i_{1}<\cdots<i_{d} \leq r$, we set

$$
\left(s ; i_{1}, i_{2}, \ldots, i_{d}\right):=\operatorname{det}\left(D\left(s ; i_{1}\right), D\left(s ; i_{2}\right), \ldots, D\left(s ; i_{d}\right)\right),
$$

which coincides with the notation for a vertex in $X_{d}\left(m, m^{\prime}\right)$ since later we identify each vertex with the minor above. 
Proof of Proposition 6.8. We shall prove the proposition in the following three steps.

Step 1. $\Delta^{L}(k ; \mathbf{i})(\tau)=\operatorname{det}(D(m ; 1), D(m ; 2), \ldots, D(m ; d))$.

It is followed from Lemma 5.3 that $\Delta^{L}(k ; \mathbf{i})(\tau)$ is given as a minor whose row (resp. column) are labeled by the set $\left\{m^{\prime}+1, \ldots, m^{\prime}+d\right\}$ (resp. $\{1, \ldots, d\}$ ) of the matrix $x^{(m)}(\tau)$. Thus, by using above notation, we have $\Delta^{L}(k ; \mathbf{i})(\tau)=\operatorname{det}\left({ }^{t}(D(m ; 1), D(m ; 2), \ldots, D(m ; d))\right)=$ $\operatorname{det}(D(m ; 1), D(m ; 2), \ldots, D(m ; d))$.

Step 2. Calculation of $\operatorname{det}(D(m ; 1), D(m ; 2), \ldots, D(m ; d))$ and labeled graph.

By using (6.2), let us calculate $\Delta^{L}(k ; \mathbf{i})(\tau)=\operatorname{det}(D(m ; 1), D(m ; 2), \ldots, D(m ; d))$ explicitly. We have

$$
\begin{aligned}
(m ; 1,2, \ldots, d)= & \operatorname{det}(D(m ; 1), D(m ; 2), \ldots, D(m ; d)) \\
=\operatorname{det}( & \frac{1}{\tau_{l_{m-1}+1}} D(m-1 ; 1)+D(m-1 ; 2), \ldots, \\
& \left.\frac{\tau_{l_{m-1}+d-1}}{\tau_{l_{m-1}+d}} D(m-1 ; d)+D(m-1 ; d+1)\right),
\end{aligned}
$$

which implies that in the notation $(6.3),(m ; 1,2, \ldots, d)$ is a linear combination of $\{(m-1$; $\left.a_{1}^{(1)}, a_{2}^{(1)}, \ldots, a_{d}^{(1)}\right) \mid 1 \leq a_{1}^{(1)}<a_{2}^{(1)}<\cdots<a_{d}^{(1)} \leq d+1, a_{i}^{(1)}=i$ or $\left.i+1\right\}$. By $(6.4)$, the coefficient of each $\left(m-1 ; a_{1}^{(1)}, a_{2}^{(1)}, \ldots, a_{d}^{(1)}\right)$ is

$$
\prod_{i=1}^{d} \frac{\tau_{l_{m-1}+a_{i}^{(1)}-1}}{\tau_{l_{m-1}+i}}
$$

which coincides with the label of the edge connecting $(m ; 1, \ldots, d)$ and $\left(m-1 ; a_{1}^{(1)}, a_{2}^{(1)}, \ldots, a_{d}^{(1)}\right)$ defined in Definition 6.4, see (6.1).

Using the formula (6.2) again, we see that each $\left(m-1 ; a_{1}^{(1)}, a_{2}^{(1)}, \ldots, a_{d}^{(1)}\right)$ is a linear combination of $\left\{\left(m-2 ; a_{1}^{(2)}, a_{2}^{(2)}, \ldots, a_{d}^{(2)}\right) \mid 1 \leq a_{1}^{(2)}<a_{2}^{(2)}<\cdots<a_{d}^{(2)} \leq d+2, a_{i}^{(2)}=a_{i}^{(1)}\right.$ or $\left.a_{i}^{(1)}+1\right\}$ in the same way as (6.4). Then, the coefficient of $\left(m-2 ; a_{1}^{(2)}, a_{2}^{(2)}, \ldots, a_{d}^{(2)}\right)$ is

$$
\prod_{i=1}^{d} \frac{\tau_{l_{m-2}+a_{i}^{(2)}-1}}{\tau_{l_{m-2}+a_{i}^{(1)}}}
$$

which coincides with the label of the edge connecting $\left(m-1 ; a_{1}^{(1)}, a_{2}^{(1)}, \ldots, a_{d}^{(1)}\right)$ and $(m-2$; $\left.a_{1}^{(2)}, a_{2}^{(2)}, \ldots, a_{d}^{(2)}\right)$. Thus $(m ; 1,2, \ldots, d)$ is a linear combination of $\left(m-2 ; a_{1}^{(2)}, a_{2}^{(2)}, \ldots, a_{d}^{(2)}\right)$, $1 \leq a_{1}^{(2)}<a_{2}^{(2)}<\cdots<a_{d}^{(2)} \leq d+2$, whose coefficient is

$$
\sum_{a_{1}^{(1)}, \ldots, a_{d}^{(1)}}\left(\prod_{i=1}^{d} \frac{\tau_{l_{m-1}+a_{i}^{(1)}-1}}{\tau_{l_{m-1}+i}} \times \prod_{i=1}^{d} \frac{\tau_{l_{m-2}+a_{i}^{(2)}-1}}{\tau_{l_{m-2}+a_{i}^{(1)}}}\right),
$$

where the index $a_{i}^{(1)}(1 \leq i \leq d)$ runs over $\left\{\left(a_{i}^{(1)}: i \in\{1, \ldots, d\}\right) \mid a_{i}^{(2)}=a_{i}^{(1)}\right.$ or $a_{i}^{(1)}+1, a_{i}^{(1)}=i$ or $\left.i+1, a_{i}^{(1)}<a_{i+1}^{(1)}\right\}$.

Repeating this argument, we see that $(m ; 1,2, \ldots, d)$ is a linear combination of $\left(0 ; a_{1}^{(m)}, a_{2}^{(m)}\right.$, $\left.\ldots, a_{d}^{(m)}\right), 1 \leq a_{1}^{(m)}<a_{2}^{(m)}<\cdots<a_{d}^{(m)} \leq m+d$, whose coefficient is

$$
\sum_{a_{i}^{(j)}, i \in\{1, \ldots, d\}, 1 \leq j \leq m-1}\left(\prod_{i=1}^{d} \frac{\tau_{l_{m-1}+a_{i}^{(1)}}-1}{\tau_{l_{m-1}+i}} \times \prod_{i=1}^{d} \frac{\tau_{l_{m-2}+a_{i}^{(2)}}-1}{\tau_{l_{m-2}+a_{i}^{(1)}}} \times \cdots \times \prod_{i=1}^{d} \frac{\tau_{l_{0}+a_{i}^{(m)}}-1}{\tau_{l_{0}+a_{i}^{(m-1)}}}\right),
$$


where the index $a_{i}^{(j)}$ runs over $\left\{\left(a_{i}^{(j)}: i \in\{1, \ldots, d\}, 1 \leq j \leq m-1\right) \mid a_{i}^{(j+1)}=a_{i}^{(j)}\right.$ or $a_{i}^{(j)}+1$, $a_{i}^{(j)}<a_{i+1}^{(j)}, a_{i}^{(1)}=i$ or $\left.i+1\right\}$.

Step 3. One-to-one correspondence between paths and terms of $\Delta^{L}(k ; \mathbf{i})(\tau)$.

Let us recall that $\left(0 ; a_{1}^{(m)}, a_{2}^{(m)}, \ldots, a_{d}^{(m)}\right)$ means a minor of the identity matrix, and $\left(0 ; a_{1}^{(m)}, a_{2}^{(m)}, \ldots, a_{d}^{(m)}\right)=\operatorname{det}\left(D\left(0 ; a_{1}^{(m)}\right), D\left(0 ; a_{2}^{(m)}\right), \ldots, D\left(0 ; a_{d}^{(m)}\right)\right)$, and each $D(0 ; i)$ is given as follows:

$$
D(0 ; i)=\left(\begin{array}{c}
x_{m^{\prime}+1, i}^{(0)} \\
\vdots \\
x_{m^{\prime}+d, i}^{(0)}
\end{array}\right)=\left(\begin{array}{c}
\delta_{m^{\prime}+1, i} \\
\vdots \\
\delta_{m^{\prime}+d, i}
\end{array}\right) .
$$

By $1 \leq a_{1}^{(m)}<a_{2}^{(m)}<\cdots<a_{d}^{(m)} \leq m+d$ (Definition 6.2), we have

$$
\left(0 ; a_{1}^{(m)}, a_{2}^{(m)}, \ldots, a_{d}^{(m)}\right)= \begin{cases}1 & \text { if } a_{1}^{(m)}=m^{\prime}+1, a_{2}^{(m)}=m^{\prime}+2, \ldots, a_{d}^{(m)}=m^{\prime}+d, \\ 0 & \text { otherwise. }\end{cases}
$$

Therefore, $\Delta^{L}(k ; \mathbf{i})(\tau)=\operatorname{det}(D(m ; 1), D(m ; 2), \ldots, D(m ; d))$ is equal to the coefficient of $\left(0 ; m^{\prime}+\right.$ $\left.1, m^{\prime}+2, \ldots, m^{\prime}+d\right)$. By $(6.5)$, setting $a_{i}^{(0)}=i, a_{i}^{(m)}=m^{\prime}+i$, we have

$$
\begin{aligned}
\Delta^{L}(k ; \mathbf{i})(\tau)= & \sum_{a_{i}^{(j)}, i \in\{1, \ldots, d\}, 1 \leq j \leq m-1}\left(\prod_{i=1}^{d} \frac{\tau_{l_{m-1}+a_{i}^{(1)}-1}}{\tau_{l_{m-1}+a_{i}^{(0)}}}\right. \\
& \left.\times \prod_{i=1}^{d} \frac{\tau_{l_{m-2}+a_{i}^{(2)}-1}}{\tau_{l_{m-2}+a_{i}^{(1)}}} \times \cdots \times \prod_{i=1}^{d} \frac{\tau_{l_{0}+a_{i}^{(m)}-1}}{\tau_{l_{0}+a_{i}^{(m-1)}}}\right),
\end{aligned}
$$

where the index $a_{i}^{(j)}$ runs over $\left\{\left(a_{i}^{(j)}: i \in\{1, \ldots, d\}, 1 \leq j \leq m-1\right) \mid a_{i}^{(j+1)}=a_{i}^{(j)}\right.$ or $a_{i}^{(j)}+1$, $\left.a_{i}^{(j)}<a_{i+1}^{(j)}, 0 \leq j \leq m-1\right\}$. Note that these conditions equal to the ones in Definition 6.2(ii) and (iii), and the conditions $a_{i}^{(0)}=i, a_{i}^{(m)}=m^{\prime}+i$ are equal to the ones in Definition 6.2(iv). We see that the summand in (6.6) is equal to the label of the path

$$
\begin{aligned}
\left(m ; a_{1}^{(0)}, \ldots, a_{d}^{(0)}\right) & \rightarrow\left(m-1 ; a_{1}^{(1)}, \ldots, a_{d}^{(1)}\right) \rightarrow\left(m-2 ; a_{1}^{(2)}, \ldots, a_{d}^{(2)}\right) \rightarrow \cdots \\
& \rightarrow\left(1 ; a_{1}^{(m-1)}, \ldots, a_{d}^{(m-1)}\right) \rightarrow\left(0 ; a_{1}^{(m)}, \ldots, a_{d}^{(m)}\right) \in X_{d}\left(m, m^{\prime}\right) .
\end{aligned}
$$

Hence we obtain $\Delta^{L}(k ; \mathbf{i})(\tau)=\sum_{p \in X_{d}\left(m, m^{\prime}\right)} Q(p)$.

Example 6.9. We use the same notations $m, m^{\prime}$ and $d=i_{k}$ at the beginning of Section 5 . We set rank $r=4, u=s_{1} s_{2} s_{3} s_{4} s_{1} s_{2} s_{3} s_{1} s_{2} s_{1} \in W$ and $k=6$, which is the same setting as in introduction. Let

$$
\mathbf{i}:=(1,2,3,4,1,2,3,1,2,1)
$$

be a reduced for $u$, and let

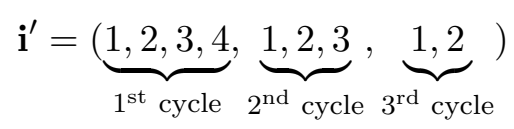

be a reduced word for $u s_{1}$. By Lemma 5.4 , we get $\Delta^{L}(6 ; \mathbf{i})(\tau)=\Delta^{L}\left(6 ; \mathbf{i}^{\prime}\right)(\tau)$. Since $m=3$, $m^{\prime}=2$ and $d:=i_{k}=2$, it follows from Example 6.5 and Proposition 6.8 that

$$
\Delta^{L}(6 ; \mathbf{i})(\tau)=\Delta^{L}\left(6 ; \mathbf{i}^{\prime}\right)(\tau)=\sum_{p \in X_{2}(3,2)} Q(p)
$$




$$
\begin{aligned}
& =\frac{1}{\tau_{l_{2}+2}}+\frac{\tau_{l_{1}+2}}{\tau_{l_{2}+1} \tau_{l_{1}+3}}+\frac{\tau_{l_{0}+3}}{\tau_{l_{2}+1} \tau_{l_{0}+4}}+\frac{\tau_{l_{1}+1}}{\tau_{l_{1}+3}}+\frac{\tau_{l_{1}+1} \tau_{l_{0}+3}}{\tau_{l_{1}+2} \tau_{l_{0}+4}}+\frac{\tau_{l_{0}+2}}{\tau_{l_{0}+4}} \\
& =\frac{1}{\tau_{9}}+\frac{\tau_{6}}{\tau_{8} \tau_{7}}+\frac{\tau_{3}}{\tau_{8} \tau_{4}}+\frac{\tau_{5}}{\tau_{7}}+\frac{\tau_{5} \tau_{3}}{\tau_{6} \tau_{4}}+\frac{\tau_{2}}{\tau_{4}}
\end{aligned}
$$

which is equal to (1.4).

\subsection{The explicit description of $\Delta^{L}(k ; i)(\tau)$}

In Proposition 6.8, we had described the terms of $\Delta^{L}(k ; \mathbf{i})(\tau)$ as the paths in $X_{d}\left(m, m^{\prime}\right)$. In this subsection, we shall describe $\Delta^{L}(k ; \mathbf{i})(\tau)$ explicitly by using some properties of paths. This description will be used in the proof of Theorem 5.6. First, we need to show some lemmas.

Let us write a path $p \in X_{d}\left(m, m^{\prime}\right)$ as follows

$$
\begin{aligned}
p= & \left(m ; a_{1}^{(0)}, \ldots, a_{d}^{(0)}\right) \rightarrow\left(m-1 ; a_{1}^{(1)}, \ldots, a_{d}^{(1)}\right) \rightarrow\left(m-2 ; a_{1}^{(2)}, \ldots, a_{d}^{(2)}\right) \rightarrow \cdots \\
& \rightarrow\left(1 ; a_{1}^{(m-1)}, \ldots, a_{d}^{(m-1)}\right) \rightarrow\left(0 ; a_{1}^{(m)}, \ldots, a_{d}^{(m)}\right) .
\end{aligned}
$$

We begin with the following lemma:

Lemma 6.10. For a path $p(6.7)$ and $i \in\{1, \ldots, d\}$, we have

$$
\#\left\{s \mid a_{i}^{(s)}=a_{i}^{(s+1)}, 0 \leq s \leq m-1\right\}=m-m^{\prime} .
$$

Proof. By Definition 6.2(iii) and (iv), we have

$$
i=a_{i}^{(0)} \leq a_{i}^{(1)} \leq \cdots \leq a_{i}^{(m)}=m^{\prime}+i, \quad a_{i}^{(s+1)}=a_{i}^{(s)} \quad \text { or } \quad a_{i}^{(s)}+1 .
$$

Thus, we get

$$
\#\left\{s \mid a_{i}^{(s+1)}=a_{i}^{(s)}+1,0 \leq s \leq m-1\right\}=m^{\prime},
$$

which implies that \#\{s|$\left.a_{i}^{(s)}=a_{i}^{(s+1)}, 0 \leq s \leq m-1\right\}=m-m^{\prime}$.

Definition 6.11. For a path $p(6.7)$ and $i \in\{1, \ldots, d\}$, we set $\left\{q_{i}^{(j)}\right\}_{1 \leq j \leq m-m^{\prime}}, 0 \leq q_{i}^{(1)}<\cdots<$ $q_{i}^{\left(m-m^{\prime}\right)} \leq m-1$, as

$$
\left\{q_{i}^{(1)}, q_{i}^{(2)}, \ldots, q_{i}^{\left(m-m^{\prime}\right)}\right\}:=\left\{q \mid a_{i}^{(q)}=a_{i}^{(q+1)}, 0 \leq q \leq m-1\right\} .
$$

We also set $k_{i}^{(j)} \in\left[1, m^{\prime}+d\right]\left(1 \leq i \leq d, 1 \leq j \leq m-m^{\prime}\right)$ as

$$
k_{i}^{(j)}:=a_{i}^{\left(q_{i}^{(j)}\right)} .
$$

\section{Lemma 6.12.}

(i) For $1 \leq i \leq d$ and $1 \leq j \leq m-m^{\prime}$,

$$
q_{i}^{(j)}=k_{i}^{(j)}+j-i-1 .
$$

(ii) For $1 \leq j \leq m-m^{\prime}$ and $1 \leq i \leq d-1$,

$$
1 \leq k_{i}^{(j)}<k_{i+1}^{(j)} \leq m^{\prime}+d, \quad q_{i}^{(j)} \leq q_{i+1}^{(j)} .
$$


Proof. (i) The definition of $q_{i}^{(j)}$ in (6.8) means that the path $p$ has the following $i$-sequence (Definition 6.6):

$$
\begin{aligned}
& a_{i}^{(0)}=i, \quad a_{i}^{(1)}=i+1, \quad a_{i}^{(2)}=i+2, \quad \ldots, \quad a_{i}^{\left(q_{i}^{(1)}\right)}=i+q_{i}^{(1)}, \\
& a_{i}^{\left(q_{i}^{(1)}+1\right)}=i+q_{i}^{(1)}, \quad a_{i}^{\left(q_{i}^{(1)}+2\right)}=i+q_{i}^{(1)}+1, \quad \ldots, \quad a_{i}^{\left(q_{i}^{(2)}\right)}=i+q_{i}^{(2)}-1, \\
& a_{i}^{\left(q_{i}^{(2)}+1\right)}=i+q_{i}^{(2)}-1, \quad a_{i}^{\left(q_{i}^{(2)}+2\right)}=i+q_{i}^{(2)}, \quad \ldots, \quad a_{i}^{\left(q_{i}^{(3)}\right)}=i+q_{i}^{(3)}-2, \\
& a_{i}^{\left(q_{i}^{(j-1)}+1\right)}=i+q_{i}^{(j-1)}-j+2, \quad a_{i}^{\left(q_{i}^{(j-1)}+2\right)}=i+q_{i}^{(j-1)}-j+3, \quad \ldots, \\
& a_{i}^{\left(q_{i}^{(j)}\right)}=i+q_{i}^{(j)}-j+1, \quad a_{i}^{\left(q_{i}^{(j)}+1\right)}=i+q_{i}^{(j)}-j+1, \quad a_{i}^{\left(q_{i}^{(j)}+2\right)}=i+q_{i}^{(j)}-j+2,
\end{aligned}
$$

Hence we have

$$
k_{i}^{(j)}=a_{i}^{\left(q_{i}^{(j)}\right)}=i+q_{i}^{(j)}-j+1,
$$

which implies $q_{i}^{(j)}=k_{i}^{(j)}+j-i-1$.

(ii) By Definition 6.2(iii),

$$
\begin{aligned}
& i=a_{i}^{(0)} \leq a_{i}^{(1)} \leq \cdots \leq a_{i}^{\left(q_{i+1}^{(j)}\right)} \leq a_{i}^{\left(q_{i+1}^{(j)}+1\right)}, \\
& a_{i}^{(\zeta)}=a_{i}^{(\zeta-1)} \quad \text { or } \quad a_{i}^{(\zeta-1)}+1, \quad 1 \leq \zeta \leq q_{i+1}^{(j)}+1 .
\end{aligned}
$$

We obtain

$$
q_{i+1}^{(j)}+1-j \geq \#\left\{\zeta \mid a_{i}^{(\zeta)}=a_{i}^{(\zeta-1)}+1,1 \leq \zeta \leq q_{i+1}^{(j)}+1\right\},
$$

otherwise, it follows from (6.11) and (i) that $a_{i}^{\left(q_{i+1}^{(j)}+1\right)}>i+q_{i+1}^{(j)}+1-j=k_{i+1}-1=a_{i+1}^{\left(q_{i+1}^{(j)}\right)}-1$, and hence $a_{i}^{\left(q_{i+1}^{(j)}+1\right)} \geq a_{i+1}^{\left(q_{i+1}^{(j)}\right)}=a_{i+1}^{\left(q_{i+1}^{(j)}+1\right)}$, which contradicts Definition 6.2(ii).

The inequality (6.12) means that

$$
j \leq \#\left\{\zeta \mid a_{i}^{(\zeta)}=a_{i}^{(\zeta-1)}, 1 \leq \zeta \leq q_{i+1}^{(j)}+1\right\} .
$$

On the other hand, using the list (6.10) (or the definition of $q_{i}^{(j)}$ in (6.8)), we have

$$
j=\#\left\{\zeta \mid a_{i}^{(\zeta)}=a_{i}^{(\zeta-1)}, 1 \leq \zeta \leq q_{i}^{(j)}+1\right\} .
$$

Since $a_{i}^{\left(q_{i}^{(j)}\right)}=a_{i}^{\left(q_{i}^{(j)}+1\right)}$, the equation (6.14) means

$$
j-1=\#\left\{\zeta \mid a_{i}^{(\zeta)}=a_{i}^{(\zeta-1)}, 1 \leq \zeta \leq q_{i}^{(j)}\right\} .
$$

Thus, by (6.13) and (6.15), we have $q_{i}^{(j)}<q_{i+1}^{(j)}+1$, and hence $q_{i}^{(j)} \leq q_{i+1}^{(j)}$, which yields $k_{i}^{(j)}<k_{i+1}^{(j)}$ since $k_{i}^{(j)}=i+q_{i}^{(j)}-j+1<i+q_{i+1}^{(j)}-j+2=(i+1)+q_{i+1}^{(j)}-j+1=k_{i+1}^{(j)}$. Remark 6.3 implies that $k_{i+1}^{(j)}:=a_{i+1}^{\left(q_{i+1}^{(j)}\right)} \leq m^{\prime}+d$.

For $1 \leq i \leq m$ and $1 \leq j \leq r$, we set the Laurent monomials

$$
\bar{C}(i, j):=\frac{\tau_{l_{i}+j-1}}{\tau_{l_{i}+j}} .
$$


Lemma 6.13. For a path $p(6.7)$, we set $q_{i}^{(j)}$ and $k_{i}^{(j)}$ as in (6.8) and (6.9). Then we have

$$
Q(p)=\prod_{i=1}^{d} \prod_{j=1}^{m-m^{\prime}} \bar{C}\left(m-q_{i}^{(j)}-1, k_{i}^{(j)}\right)
$$

Proof. Let us recall the definition of $Q(p)$ (Definition 6.4(ii)):

$$
Q(p)=\prod_{s=0}^{m-1}\left(\prod_{i=1}^{d} \frac{\tau_{l_{m-s-1}+a_{i}^{(s+1)}-1}}{\tau_{l_{m-s-1}+a_{i}^{(s)}}}\right)
$$

For each $i=1,2, \ldots, d$, the $i$-sequence

$$
i=a_{i}^{(0)} \leq a_{i}^{(1)} \leq a_{i}^{(2)} \leq \cdots \leq a_{i}^{(m)}=m^{\prime}+i
$$

of $p$ satisfies $a_{i}^{(s+1)}=a_{i}^{(s)}$ or $a_{i}^{(s)}+1,0 \leq s \leq m-1$, by Definition 6.2(iii). If $a_{i}^{(s+1)}=a_{i}^{(s)}+1$, then

$$
\frac{\tau_{l_{m-s-1}+a_{i}^{(s+1)}-1}}{\tau_{l_{m-s-1}+a_{i}^{(s)}}}=1 .
$$

Therefore, it follows from the definition (6.8) of $q_{i}^{(j)}$ and $k_{i}^{(j)}:=a_{i}^{\left(q_{i}^{(j)}\right)}=a_{i}^{\left(q_{i}^{(j)}+1\right)}$ that

$$
\prod_{s=0}^{m-1} \frac{\tau_{l_{m-s-1}+a_{i}^{(s+1)}-1}}{\tau_{l_{m-s-1}+a_{i}^{(s)}}}=\prod_{j=1}^{m-m^{\prime}} \frac{\tau_{l}{ }_{m-q_{i}^{(j)}-1}+k_{i}^{(j)}-1}{\tau_{l_{m-q_{i}}(j)}+k_{i}^{(j)}}=\prod_{j=1}^{m-m^{\prime}} \bar{C}\left(m-q_{i}^{(j)}-1, k_{i}^{(j)}\right),
$$

which implies (6.17) by (6.18).

Let us describe $\Delta^{L}(k ; \mathbf{i})(\tau)$ explicitly.

\section{Proposition 6.14.}

$$
\Delta^{L}(k ; \mathbf{i})(\tau)=\sum_{(*)} \prod_{i=1}^{d} \prod_{j=1}^{m-m^{\prime}} \bar{C}\left(m-K_{i}^{(j)}-j+i, K_{i}^{(j)}\right) .
$$

where $(*)$ is the conditions for $K_{i}^{(j)}\left(1 \leq i \leq d, 1 \leq j \leq m-m^{\prime}\right): 1 \leq K_{1}^{(j)}<K_{2}^{(j)}<\cdots<$ $K_{d}^{(j)} \leq m^{\prime}+d, 1 \leq j \leq m-m^{\prime}, i \leq K_{i}^{(1)} \leq K_{i}^{(2)} \leq \cdots \leq K_{i}^{\left(m-m^{\prime}\right)} \leq m^{\prime}+i, 1 \leq i \leq d$.

Proof. Using Lemmas 6.12(i) and 6.13, we see that $Q(p), p \in X_{d}\left(m, m^{\prime}\right)$, is described as

$$
Q(p)=\prod_{i=1}^{d} \prod_{j=1}^{m-m^{\prime}} \bar{C}\left(m-k_{i}^{(j)}-j+i, k_{i}^{(j)}\right)
$$

with $\left\{k_{i}^{(j)}\right\}_{1 \leq i \leq d, 1 \leq j \leq m-m^{\prime}}$ which satisfy the conditions in Lemma 6.12 (ii), that is, $1 \leq k_{1}^{(j)}<$ $k_{2}^{(j)}<\cdots<k_{d}^{(\bar{j})} \leq m^{\prime}+d$. Furthermore, Definition 6.2(iii) and (iv) show that $i=a_{i}^{(0)} \leq a_{i}^{(1)} \leq$ $\cdots \leq a_{i}^{(m)}=m^{\prime}+i$, which means that $i \leq k_{i}^{(1)} \leq k_{i}^{(2)} \leq \cdots \leq k_{i}^{\left(m-m^{\prime}\right)} \leq m^{\prime}+i$ for $1 \leq i \leq d$ by $k_{i}^{(j)}:=a_{i}^{\left(q_{i}^{(j)}\right)}$ and $q_{i}^{(j)}<q_{i}^{(j+1)}$ (see Definition 6.11). Thus, $\left\{k_{i}^{(j)}\right\}$ satisfies the conditions $(*)$ in Proposition 6.14. 
Conversely, let $\left\{K_{i}^{(j)}\right\}_{1 \leq i \leq d, 1 \leq j \leq m-m^{\prime}}$ the set of numbers which satisfies the conditions $(*)$ in Proposition 6.14:

$$
1 \leq K_{1}^{(j)}<K_{2}^{(j)}<\cdots<K_{d}^{(j)} \leq m^{\prime}+d, \quad 1 \leq j \leq m-m^{\prime}
$$

and

$$
i \leq K_{i}^{(1)} \leq \cdots \leq K_{i}^{\left(m-m^{\prime}\right)} \leq m^{\prime}+i, \quad 1 \leq i \leq d .
$$

We set

$$
Q_{i}^{(j)}:=K_{i}^{(j)}+j-i-1
$$

for $1 \leq i \leq d$ and $1 \leq j \leq m-m^{\prime}$. We need to show that there exists a path $p \in X_{d}\left(m, m^{\prime}\right)$ such that

$$
Q(p)=\prod_{i=1}^{d} \prod_{j=1}^{m-m^{\prime}} \bar{C}\left(m-Q_{i}^{(j)}-1, K_{i}^{(j)}\right)=\prod_{i=1}^{d} \prod_{j=1}^{m-m^{\prime}} \bar{C}\left(m-K_{i}^{(j)}-j+i, K_{i}^{(j)}\right) .
$$

Since we supposed $K_{i}^{(j)}<K_{i+1}^{(j)}$, we can easily verify

$$
Q_{i}^{(j)} \leq Q_{i+1}^{(j)}
$$

by (6.20). We claim that $0 \leq Q_{i}^{(j)} \leq m-1$ for $1 \leq i \leq d$ and $1 \leq j \leq m-m^{\prime}$. By the condition (6.19), we get $i \leq K_{i}^{(j)}$. So it is clear that $0 \leq Q_{i}^{(j)}$. It follows from the condition (6.19) and (6.20) that $Q_{i}^{(j)}=K_{i}^{(j)}+j-i-1 \leq m^{\prime}+i+j-i-1=m^{\prime}+j-1 \leq m-1$. Therefore, we have $0 \leq Q_{i}^{(j)} \leq m-1$ for all $1 \leq i \leq d$ and $1 \leq j \leq m-m^{\prime}$.

We define a path $p=\left(m ; a_{1}^{(0)}, \ldots, a_{d}^{(0)}\right) \rightarrow \cdots \rightarrow\left(0 ; a_{1}^{(m)}, \ldots, a_{d}^{(m)}\right) \in X_{d}\left(m, m^{\prime}\right)$ as follows. For $i, 1 \leq i \leq d$, we define the $i$-sequence (Definition 6.6) of $p$ as

$$
\begin{aligned}
& a_{i}^{(0)}=i, \quad a_{i}^{(1)}=i+1, \quad a_{i}^{(2)}=i+2, \quad \ldots, \quad a_{i}^{\left(Q_{i}^{(1)}\right)}=i+Q_{i}^{(1)}, \\
& a_{i}^{\left(Q_{i}^{(1)}+1\right)}=i+Q_{i}^{(1)}, \quad a_{i}^{\left(Q_{i}^{(1)}+2\right)}=i+Q_{i}^{(1)}+1, \quad \ldots, \quad a_{i}^{\left(Q_{i}^{(2)}\right)}=i+Q_{i}^{(2)}-1, \\
& a_{i}^{\left(Q_{i}^{(2)}+1\right)}=i+Q_{i}^{(2)}-1, \quad a_{i}^{\left(Q_{i}^{(2)}+2\right)}=i+Q_{i}^{(2)}, \quad \ldots, \quad a_{i}^{\left(Q_{i}^{(3)}\right)}=i+Q_{i}^{(3)}-2, \\
& \ldots \ldots \ldots \ldots \ldots \ldots \ldots \ldots \ldots \ldots \ldots \ldots \ldots \ldots \ldots \ldots \\
& a_{i}^{\left(Q_{i}^{\left(m-m^{\prime}-1\right)}+1\right)}=i+Q_{i}^{\left(m-m^{\prime}-1\right)}-m+m^{\prime}+2, \quad \ldots, \\
& a_{i}^{\left(Q_{i}^{\left(m-m^{\prime}\right)}\right)}=i+Q_{i}^{\left(m-m^{\prime}\right)}-m+m^{\prime}+1, \quad a_{i}^{\left(Q_{i}^{\left(m-m^{\prime}\right)}+1\right)}=i+Q_{i}^{\left(m-m^{\prime}\right)}-m+m^{\prime}+1, \\
& a_{i}^{\left(Q_{i}^{\left(m-m^{\prime}\right)}+2\right)}=i+Q_{i}^{\left(m-m^{\prime}\right)}-m+m^{\prime}+2, \\
& a_{i}^{\left(Q_{i}^{\left(m-m^{\prime}\right)}+3\right)}=i+Q_{i}^{\left(m-m^{\prime}\right)}-m+m^{\prime}+3, \quad \ldots, \quad a_{i}^{(m)}=m^{\prime}+i .
\end{aligned}
$$

It is easy to see that $a_{i}^{\left(Q_{i}^{(j)}\right)}=K_{i}^{(j)}, 1 \leq j \leq m-m^{\prime}$, by (6.20) and (6.23). Clearly, the path $p$ satisfies Definition 6.2(iii) and (iv). For $1 \leq s \leq m$, we obtain $a_{i}^{(s)}<a_{i+1}^{(s)}$ by (6.22) and (6.23).

Hence $p$ is well-defined, and (6.21) is follows from Lemma 6.13 (see (6.8) and (6.9)). Thus, Proposition 6.14 follows from Proposition 6.8. 
Example 6.15. We use the same setting in Example 6.9. Since $m=3, m^{\prime}=2$ and $d:=i_{k}=2$, it follows from Proposition 6.14 that

$$
\begin{aligned}
\Delta^{L}(6 ; \mathbf{i})(\tau)= & \sum_{1 \leq K_{1}<K_{2} \leq 4}\left(\prod_{i=1}^{2} \bar{C}\left(i-K_{i}+2, K_{i}\right)\right) \\
= & \bar{C}(2,1) \bar{C}(2,2)+\bar{C}(2,1) \bar{C}(1,3)+\bar{C}(2,1) \bar{C}(0,4) \\
& +\bar{C}(1,2) \bar{C}(1,3)+\bar{C}(1,2) \bar{C}(0,4)+\bar{C}(0,3) \bar{C}(0,4) \\
= & \frac{1}{\tau_{l_{2}+2}}+\frac{\tau_{l_{1}+2}}{\tau_{l_{2}+1} \tau_{l_{1}+3}}+\frac{\tau_{l_{0}+3}}{\tau_{l_{2}+1} \tau_{l_{0}+4}}+\frac{\tau_{l_{1}+1}}{\tau_{l_{1}+3}}+\frac{\tau_{l_{1}+1} \tau_{l_{0}+3}}{\tau_{l_{1}+2} \tau_{l_{0}+4}}+\frac{\tau_{l_{0}+2}}{\tau_{l_{0}+4}}
\end{aligned}
$$

which is equal to the one in Example 6.9.

\subsection{The completion of the proof of Theorem 5.6}

In this subsection, we shall complete the proof of Theorem 5.6.

Let us recall the definition (3.3) of $A_{s, i}$. Since we identify the variables $\left\{Y_{s, i}\right\}$ with $\left\{\tau_{l_{s}+i}\right\}$, we have the following (see Remark 3.7)

$$
A_{s, i}=\frac{\tau_{l_{s}+i} \tau_{l_{s+1}+i}}{\tau_{l_{s}+i+1} \tau_{l_{s+1}+i-1}}, \quad 0 \leq s \leq m-1, \quad 1 \leq i \leq r .
$$

Therefore, by the definition $(6.16)$ of $\bar{C}(s, i)$, we have

$$
\bar{C}(s, i) \cdot A_{s-1, i}=\bar{C}(s-1, i+1) .
$$

Lemma 6.16. For a path $p \in X_{d}\left(m, m^{\prime}\right)$, we describe the monomial $Q(p)$ as in Lemma 6.13

$$
Q(p)=\prod_{i=1}^{d} \prod_{j=1}^{m-m^{\prime}} \bar{C}\left(m-K_{i}^{(j)}-j+i, K_{i}^{(j)}\right)
$$

where $\left\{K_{i}^{(j)}\right\}$ satisfies the condition $(*)$ in Proposition 6.14. For $s, 1 \leq s<m^{\prime}+d$, if $\tilde{e}_{s} Q(p) \neq 0$, then there exist $i$ and $j, 1 \leq i \leq d, 1 \leq j \leq m-m^{\prime}$, such that

$$
K_{i}^{(j)}=s \quad \text { and } \quad \tilde{e}_{s} Q(p)=Q(p) \cdot A_{m-K_{i}^{(j)}-j+i-1, s} .
$$

Furthermore, there exists a path $p^{\prime} \in X_{d}\left(m, m^{\prime}\right)$ such that

$$
\tilde{e}_{s} Q(p)=Q\left(p^{\prime}\right) .
$$

Proof. We suppose that the monomial $Q(p)$ does not include factor as in the form $\tau_{l_{t}+s}^{-1}, 0 \leq t \leq$ $m-1$, which means wt $(Q(p))\left(h_{i}\right)=\varphi_{i}(Q(p))$. Hence $\varepsilon_{i}(Q(p)):=\varphi_{i}(Q(p))-\operatorname{wt}(Q(p))\left(h_{i}\right)=0$, which contradicts the assumption $\tilde{e}_{s} Q(p) \neq 0$. Hence the monomial $Q(p)$ includes factor $\tau_{l_{t}+s}^{-1}$, $0 \leq t \leq m-1$. We set the numbers $0 \leq t_{1}<t_{2}<\cdots<t_{\xi} \leq m-1$ by

$$
\left\{t_{1}, t_{2}, \ldots, t_{\xi}\right\}:=\left\{t \mid Q(p) \text { includes factors } \tau_{l_{t}+s}^{-1}\right\}, \quad 1 \leq \xi .
$$

The definition (6.16) of $\bar{C}(t, s)$ and (6.25) show that there exist $1 \leq i_{1}, \ldots, i_{\xi} \leq d$ and $1 \leq$ $j_{1}, \ldots, j_{\xi} \leq m-m^{\prime}$ such that

$$
K_{i_{a}}^{\left(j_{a}\right)}=s \quad \text { and } \quad t_{a}=m-K_{i_{a}}^{\left(j_{a}\right)}-j_{a}+i_{a}, \quad a=1,2, \ldots, \xi .
$$


As in Example 3.9, for a given $s \in I$ and monomial $Y=\prod_{q \in \mathbb{Z}, i \in I} \tau_{l_{q}+i}^{\zeta_{q, i}}$, we define $\nu_{Y}(n):=$ $\sum_{q \leq n} \zeta_{q, s}, n \in \mathbb{Z}$

We set $Y:=Q(p)$. We claim that $n_{e_{s}} \in\left\{t_{1}-1, \ldots, t_{\xi}-1\right\}(3.4)$, otherwise, we get $n_{e_{s}} \notin$ $\left\{t_{1}-1, \ldots, t_{\xi}-1\right\}$. Then $\varphi_{s}(Y)=\nu_{Y}\left(n_{e_{s}}\right) \leq \nu_{Y}\left(n_{e_{s}}+1\right)$. If $\nu_{Y}\left(n_{e_{s}}\right)<\nu_{Y}\left(n_{e_{s}}+1\right)$, then it contradicts the definition of

$$
\varphi_{s}(Y):=\max \left\{\nu_{Y}(n) \mid n \in \mathbb{Z}\right\} .
$$

If $\nu_{Y}\left(n_{e_{s}}\right)=\nu_{Y}\left(n_{e_{s}}+1\right)$, then it contradicts the definition of $n_{e_{s}}:=\max \left\{n \mid \varphi_{s}(Y)=\nu_{Y}(n)\right\}$. Hence we obtain $n_{e_{s}} \in\left\{t_{1}-1, \ldots, t_{\xi}-1\right\}$, which implies there exists $a, 1 \leq a \leq \xi$, such that $n_{e_{s}}=t_{a}-1$. By (6.28), we have $n_{e_{s}}=t_{a}-1=m-K_{i_{a}}^{\left(j_{a}\right)}-j_{a}+i_{a}-1$. Therefore, we obtain $\tilde{e}_{s} Y:=Y \cdot A_{n_{e_{s}, s}}=Y \cdot A_{m-K_{i_{a}}^{\left(j_{a}\right)}-j_{a}+i_{a}-1, s}$.

Since $Q(p)$ includes the factor $\tau_{l_{a}+s}^{-1}$, the path $p$ includes the following edge

$$
\begin{aligned}
\left(t_{a}+\right. & \left.1 ; a_{1}^{\left(m-t_{a}-1\right)}, \cdots, a_{q-1}^{\left(m-t_{a}-1\right)}, \underset{q^{\mathrm{th}}}{s}, a_{q+1}^{\left(m-t_{a}-1\right)}, \cdots, a_{d}^{\left(m-t_{a}-1\right)}\right) \\
& \rightarrow\left(t_{a} ; a_{1}^{\left(m-t_{a}\right)}, \cdots, a_{q-1}^{\left(m-t_{a}\right)}, \underset{q^{\mathrm{th}}}{s}, a_{q+1}^{\left(m-t_{a}\right)}, \cdots, a_{d}^{\left(m-t_{a}\right)}\right) \\
& \rightarrow\left(t_{a}-1 ; a_{1}^{\left(m-t_{a}+1\right)}, \cdots, a_{q-1}^{\left(m-t_{a}+1\right)}, a_{q}^{\left(m-t_{a}+1\right)}, a_{q+1}^{\left(m-t_{a}+1\right)}, \cdots, a_{d}^{\left(m-t_{a}+1\right)}\right)
\end{aligned}
$$

for some $1 \leq q \leq d$. We claim that $a_{q+1}^{\left(m-t_{a}\right)}>s+1$, otherwise $a_{q+1}^{\left(m-t_{a}\right)}=s+1$ and $a_{q+1}^{\left(m-t_{a}-1\right)}=$ $s+1$ by Definition 6.2(ii) and (iii), which implies that $Q(p)$ does not include the factor $\tau_{l_{t_{a}}+s}^{-1}$ by Definition 6.4(i). It contradicts the definition of $t_{a}$. So we get

$$
a_{q+1}^{\left(m-t_{a}\right)}>s+1 .
$$

If $t_{a}=0$, then Definition 6.2(iv) means that $s=m^{\prime}+q$ (by the assumption $s<m^{\prime}+d$, we get $q<d$ ), and $a_{q+1}^{\left(m-t_{a}\right)}=m^{\prime}+q+1=s+1$, which contradicts (6.30). So we have $1 \leq t_{a}$. We also claim that $a_{q}^{\left(m-t_{a}+1\right)}>s$, otherwise $a_{q}^{\left(m-t_{a}+1\right)}=s$, which implies that $Q(p)$ includes the factor $\tau_{l_{t_{a}+1}+s}^{-1}$ by $a_{q+1}^{\left(m-t_{a}+1\right)} \geq a_{q+1}^{\left(m-t_{a}\right)}>s+1$. Therefore, $\varphi_{s}(Y)=\nu_{Y}\left(n_{e_{s}}\right)=\nu_{Y}\left(t_{a}-1\right)<\nu_{Y}\left(t_{a}-2\right)$, which contradicts (6.29). So we get $a_{q}^{\left(m-t_{a}+1\right)}>s$, which means

$$
a_{q}^{\left(m-t_{a}+1\right)}=s+1,
$$

by Definition 6.2(iii).

Let $p^{\prime} \in X_{d}\left(m, m^{\prime}\right)$ be the path obtained from $p$ by replacing the vertex

$$
\left(t_{a} ; a_{1}^{\left(m-t_{a}\right)}, \ldots, a_{q-1}^{\left(m-t_{a}\right)}, \underset{q^{\mathrm{th}}}{s}, a_{q+1}^{\left(m-t_{a}\right)}, \ldots, a_{d}^{\left(m-t_{a}\right)}\right)
$$

by

$$
\left(t_{a} ; a_{1}^{\left(m-t_{a}\right)}, \ldots, a_{q-1}^{\left(m-t_{a}\right)}, s+1, a_{q+1}^{\left(m-t_{a}\right)}, \ldots, a_{d}^{\left(m-t_{a}\right)}\right) .
$$

By (6.30) and (6.31), the path $p^{\prime}$ is well-defined. Definition 6.4(i) shows that

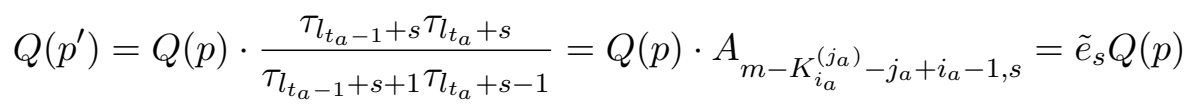

by $(6.28)$. 
Next lemma shows that the coefficients $c(b)$ in Theorem 5.6 are equal to 1 for all $b \in$ $B_{u_{\leq k}}^{-}\left(\left(m^{\prime}-m\right) \Lambda_{d}\right)$ by Proposition 6.8.

Lemma 6.17. For paths $p, p^{\prime} \in X_{d}\left(m, m^{\prime}\right)$, if $p \neq p^{\prime}$ then $Q(p) \neq Q\left(p^{\prime}\right)$.

Proof. We suppose that $Q(p)=Q\left(p^{\prime}\right)$. Let us prove $p=p^{\prime}$. We denote $p$ and $p^{\prime}$ by

$$
p=\left(m ; a_{1}^{(0)}, \ldots, a_{d}^{(0)}\right) \rightarrow \cdots \rightarrow\left(0 ; a_{1}^{(m)}, \ldots, a_{d}^{(m)}\right)
$$

and

$$
p^{\prime}=\left(m ; b_{1}^{(0)}, \ldots, b_{d}^{(0)}\right) \rightarrow \cdots \rightarrow\left(0 ; b_{1}^{(m)}, \ldots, b_{d}^{(m)}\right) .
$$

Since $Q(p)$ is the product of the labels

$$
\prod_{i=1}^{d} \frac{\tau_{l_{m-s-1}+a_{i}^{(s+1)}-1}}{\tau_{l_{m-s-1}+a_{i}^{(s)}}}
$$

of the edges $\left(m-s ; a_{1}^{(s)}, \ldots, a_{d}^{(s)}\right) \rightarrow\left(m-s-1 ; a_{1}^{(s+1)}, \ldots, a_{d}^{(s+1)}\right), 0 \leq s \leq m-1$, the assumption $Q(p)=Q\left(p^{\prime}\right)$ means that

$$
\prod_{i=1}^{d} \frac{\tau_{l_{m-s-1}+a_{i}^{(s+1)}-1}}{\tau_{l_{m-s-1}+a_{i}^{(s)}}}=\prod_{i=1}^{d} \frac{\tau_{l_{m-s-1}+b_{i}^{(s+1)}-1}}{\tau_{l_{m-s-1}+b_{i}^{(s)}}}
$$

for all $0 \leq s \leq m-1$. For $s=0$, by Definition 6.2 (iv), we get $a_{i}^{(0)}=b_{i}^{(0)}=i, 1 \leq i \leq d$. It follows from $a_{1}^{(1)}<a_{2}^{(1)}<\cdots<a_{d}^{(1)}, b_{1}^{(1)}<b_{2}^{(1)}<\cdots<b_{d}^{(1)}$ and (6.32) for $s=0$ that $a_{i}^{(1)}=b_{i}^{(1)}$, $1 \leq i \leq d$. Repeating this argument for $s=1,2,3, \ldots, m-1$, we get $a_{i}^{(s)}=b_{i}^{(s)}, 1 \leq i \leq d$, which means that $p=p^{\prime}$.

Proof of Theorem 5.6. We set

$$
\mathbb{B}:=\left\{Q(p) \mid p \in X_{d}\left(m, m^{\prime}\right)\right\}=\left\{\prod_{i=1}^{d} \prod_{j=1}^{m-m^{\prime}} \bar{C}\left(R_{i}^{(j)}, K_{i}^{(j)}\right) \mid R_{i}^{(j)}:=m-K_{i}^{(j)}-j+i\right\},
$$

where $\left\{K_{i}^{(j)}\right\}$ satisfies the conditions

$$
\begin{aligned}
& 1 \leq K_{1}^{(j)}<K_{2}^{(j)}<\cdots<K_{d}^{(j)} \leq m^{\prime}+d, \quad 1 \leq j \leq m-m^{\prime} \\
& i \leq K_{i}^{(1)} \leq \cdots \leq K_{i}^{\left(m-m^{\prime}\right)} \leq m^{\prime}+i, \quad 1 \leq i \leq d .
\end{aligned}
$$

By Proposition 6.14 and Lemma 6.17, we need to show that

$$
\mathbb{B}=\mu_{Y}\left(B_{u_{\leq k}}^{-}\left(\left(m^{\prime}-m\right) \Lambda_{d}\right)\right),
$$

where $\mu_{Y}(x)$ is an embedding of $x \in B_{u_{\leq k}}^{-}\left(\left(m^{\prime}-m\right) \Lambda_{d}\right)$ in Theorem 5.6, and

$$
Y:=\frac{1}{\tau_{l_{m-1}+d} \tau_{l_{m-2}+d} \cdots \tau_{l_{m^{\prime}}+d}}
$$

which is the lowest weight vector in $\mu_{Y}\left(B_{u_{\leq k}}^{-}\left(\left(m^{\prime}-m\right) \Lambda_{d}\right)\right)$ (Theorem 3.2(ii)). 
First, let us prove the inclusion $\mu_{Y}\left(B_{u_{\leq k}}^{-}\left(\left(m^{\prime}-m\right) \Lambda_{d}\right)\right) \subset \mathbb{B}$. Using $\bar{C}(a, b)$, the monomial $Y$ in (6.34) is described as follows

$$
\begin{aligned}
& \bar{C}(m-1,1) \cdot \bar{C}(m-1,2) \cdots \bar{C}(m-1, d) \\
& Y=\cdot \bar{C}(m-2,1) \cdot \bar{C}(m-2,2) \cdots \bar{C}(m-2, d) \\
& \text { - } \bar{C}\left(m^{\prime}, 1\right) \quad \cdot \quad \bar{C}\left(m^{\prime}, 2\right) \quad \ldots \quad \bar{C}\left(m^{\prime}, d\right) .
\end{aligned}
$$

Thus, we see that $Y \in \mathbb{B}$. It follows from Theorem 3.12 and the definition (4.3) of $u_{\leq k}$ that

$$
\begin{aligned}
& \mu_{Y}\left(B_{u_{\leq k}}^{-}\left(\left(m^{\prime}-m\right) \Lambda_{d}\right)\right)=\{\underbrace{\tilde{e}_{1}^{\left(N_{1}\left(m^{\prime}\right)\right)} \cdots \tilde{e}_{r}^{\left(N_{r}\left(m^{\prime}\right)\right)}}_{1^{\text {st }} \text { cycle }} \underbrace{\tilde{e}_{1}^{\left(N_{1}\left(m^{\prime}-1\right)\right)} \cdots \tilde{e}_{r-1}^{\left(N_{r-1}\left(m^{\prime}-1\right)\right)}}_{2^{\text {nd }} \text { cycle }} \\
& \cdots \underbrace{\tilde{e}_{1}^{\left(N_{1}(2)\right)} \cdots \tilde{e}_{r-m+2}^{\left(N_{r-m+2}(2)\right)}}_{\left(m^{\prime}-1\right)^{\text {th }} \text { cycle }} \underbrace{\tilde{e}_{1}^{\left(N_{1}(1)\right)} \ldots \tilde{e}_{d}^{\left(N_{d}(1)\right)}}_{m^{\text {th }} \text { cycle }} \cdot Y \mid N_{i}(j) \in \mathbb{Z}_{\geq 0}\} \backslash\{0\} .
\end{aligned}
$$

By (6.27), for any monomial $Z \in \mathbb{B}$ and $1 \leq s<m^{\prime}+d$, we have

$$
\tilde{e}_{s} Z \in \mathbb{B} \cup\{0\} .
$$

For an arbitrary set $\left\{N_{i}(1)\right\}_{i=1, \ldots, d}$ of non negative integers, the monomial $\tilde{e}_{1}^{\left(N_{1}(1)\right)} \cdots \tilde{e}_{d}^{\left(N_{d}(1)\right)} \cdot Y$ does not include factors in the form $\bar{C}(*, a), d+2 \leq a \leq r-m+2$, by (6.24) and (6.26). Therefore, $\tilde{e}_{a} \cdot \tilde{e}_{1}^{\left(N_{1}(1)\right)} \cdots \tilde{e}_{d}^{\left(N_{d}(1)\right)} \cdot Y=0$ for $d+2 \leq a \leq r-m+2$ by (6.26). Similarly, for an arbitrary set $\left\{N_{i}(2)\right\}_{i=1, \ldots, r-m+2}$ of non negative integers, the monomial $\tilde{e}_{1}^{\left(N_{1}(2)\right)} \cdots \tilde{e}_{r-m+2}^{\left(N_{r-m+2}(2)\right)} \tilde{e}_{1}^{\left(N_{1}(1)\right)}$ $\cdots \tilde{e}_{d}^{\left(N_{d}(1)\right)} \cdot Y$ does not include factors in the form $\bar{C}(*, a), d+3 \leq a \leq r-m+3$, which means that $\tilde{e}_{a} \cdot \tilde{e}_{1}^{\left(N_{1}(2)\right)} \ldots \tilde{e}_{r-m+2}^{\left(N_{r-m+2}(2)\right)} \tilde{e}_{1}^{\left(N_{1}(1)\right)} \ldots \tilde{e}_{d}^{\left(N_{d}(1)\right)} \cdot Y=0$.

Repeating this argument, we obtain

$$
\begin{aligned}
& \mu_{Y}\left(B_{u_{\leq k}}^{-}\left(\left(m^{\prime}-m\right) \Lambda_{d}\right)\right)=\{\underbrace{\tilde{e}_{1}^{\left(N_{1}\left(m^{\prime}\right)\right)} \cdots \tilde{e}_{m^{\prime}+d-1}^{\left(N_{m^{\prime}+d-1}\left(m^{\prime}\right)\right)}}_{1^{\text {st }} \text { cycle }} \underbrace{\tilde{e}_{1}^{\left(N_{1}\left(m^{\prime}-1\right)\right)} \cdots \tilde{e}_{m^{\prime}+d-2}^{\left(N_{m^{\prime}+d-2}\left(m^{\prime}-1\right)\right)}}_{2^{\text {nd }} \text { cycle }} \\
& \cdots \underbrace{\tilde{e}_{1}^{\left(N_{1}(2)\right)} \cdots \tilde{e}_{d+1}^{\left(N_{d+1}(2)\right)}}_{\left(m^{\prime}-1\right)^{\text {th }} \text { cycle }} \underbrace{\tilde{e}_{1}^{\left(N_{1}(1)\right)} \cdots \tilde{e}_{d}^{\left(N_{d}(1)\right)}}_{m^{\text {th }} \text { cycle }} \cdot Y \mid N_{i}(j) \in \mathbb{Z}_{\geq 0}\} \backslash\{0\} .
\end{aligned}
$$

Therefore, we get $\mu_{Y}\left(B_{u_{\leq k}}^{-}\left(\left(m^{\prime}-m\right) \Lambda_{d}\right)\right) \subset \mathbb{B}$ by $(6.35)$.

Next, we shall prove $\mathbb{B} \subset \mu_{Y}\left(B_{u_{\leq k}}^{-}\left(\left(m^{\prime}-m\right) \Lambda_{d}\right)\right)$. For this, we take an arbitrary element

$$
M:=\prod_{i=1}^{d} \prod_{j=1}^{m-m^{\prime}} \bar{C}\left(R_{i}^{(j)}, K_{i}^{(j)}\right) \in \mathbb{B} .
$$

We need to show that there exists a set $\left\{N_{i}(s)\right\}$ of non negative integers such that

$$
\begin{gathered}
\underbrace{\hat{e}_{2^{\text {nd }}}^{\left(N_{1}\left(m^{\prime}-1\right)\right)} \cdots \tilde{e}_{m^{\prime}+d-2}^{\left(N_{m^{\prime}+d-2}\left(m^{\prime}-1\right)\right)}}_{1^{\text {st }} \text { cycle }^{\left(N_{1}\left(m^{\prime}\right)\right)} \cdots \tilde{e}_{m^{\prime}+d-1}^{\left(N_{m^{\prime}+d-1}\left(m^{\prime}\right)\right)}} \\
\cdots \underbrace{\tilde{e}_{1}^{\left(N_{1}(2)\right)} \cdots \tilde{e}_{d+1}^{\left(N_{d+1}(2)\right)}}_{\left(m^{\prime}-1\right)^{\text {th }} \text { cycle }} \underbrace{\hat{e}_{1}^{\left(N_{1}(1)\right)} \cdots \tilde{e}_{d}^{\left(N_{d}(1)\right)}}_{m^{\text {th }} \text { cycle }} \cdot Y=M .
\end{gathered}
$$


We set $\left\{N_{i}(s)\right\}$ as follows (see Example 6.18). First, we set $\left\{N_{i}(1)\right\}_{i=1,2, \ldots, d}$ as

$$
\begin{aligned}
& N_{d}(1):=\#\left\{1 \leq j \leq m-m^{\prime} \mid K_{d}^{(j)}-d=m^{\prime}\right\}, \\
& N_{d-1}(1):=\#\left\{1 \leq j \leq m-m^{\prime} \mid K_{d-1}^{(j)}-(d-1)=m^{\prime}\right\}, \\
& N_{d-2}(1):=\#\left\{1 \leq j \leq m-m^{\prime} \mid K_{d-2}^{(j)}-(d-2)=m^{\prime}\right\}, \\
& \cdots \cdots \cdots \cdots \cdots \cdots \cdots \cdots \cdots \cdots \cdots \cdots \cdots \cdots \cdots \cdots \cdots \cdots \cdots \cdots \cdots \cdots \\
& N_{1}(1):=\#\left\{1 \leq j \leq m-m^{\prime} \mid K_{1}^{(j)}-1=m^{\prime}\right\} .
\end{aligned}
$$

Note that since $K_{1}^{(j)}<\cdots<K_{d}^{(j)}$, we have $N_{1}(1)<\cdots<N_{d}(1)$. As seen in Example 3.9, by applying $\tilde{e}_{d}^{\left(N_{d}(1)\right)}$ to $Y$, the factors $\bar{C}\left(m^{\prime}, d\right), \bar{C}\left(m^{\prime}+1, d\right), \ldots, \bar{C}\left(m^{\prime}+N_{d}(1)-1, d\right)$ in $Y$ turn out $\bar{C}\left(m^{\prime}-1, d+1\right), \bar{C}\left(m^{\prime}, d+1\right), \ldots, \bar{C}\left(m^{\prime}+N_{d}(1)-2, d+1\right)$ by Remark 3.4, (6.24) and (6.26). Then the monomial $\tilde{e}_{d}^{\left(N_{d}(1)\right)} \cdot Y$ does not include factors in the form $\tau_{l_{q}+d-1}, 0 \leq q \leq m-1$. Thus, we can use Example 3.9 again when we apply $\tilde{e}_{d-1}^{\left(N_{d-1}(1)\right)}$ to $\left(\tilde{e}_{d}^{\left(N_{d}(1)\right)} \cdot Y\right)$. Then the factors $\bar{C}\left(m^{\prime}, d-1\right), \bar{C}\left(m^{\prime}+1, d-1\right), \ldots, \bar{C}\left(m^{\prime}+N_{d-1}(1)-1, d-1\right)$ in $\left(\tilde{e}_{d}^{\left(N_{d}(1)\right)} \cdot Y\right)$ turn out $\bar{C}\left(m^{\prime}-1, d\right)$, $\bar{C}\left(m^{\prime}, d\right), \ldots, \bar{C}\left(m^{\prime}+N_{d-1}(1)-2, d\right)$, and the monomial $\tilde{e}_{d-1}^{\left(N_{d-1}(1)\right)} \tilde{e}_{d}^{\left(N_{d}(1)\right)} \cdot Y$ does not include factors in the form $\tau_{l_{q}+d-2}, 0 \leq q \leq m-1$. After all, by using Example 3.9 repeatedly, we see that the monomial $\tilde{e}_{1}^{\left(N_{1}(1)\right)} \ldots \tilde{e}_{d-1}^{\left(N_{d-1}(1)\right)} \tilde{e}_{d}^{\left(N_{d}(1)\right)} \cdot Y$ is obtained from $Y$ by replacing $\bar{C}\left(m^{\prime}, \zeta\right)$, $\bar{C}\left(m^{\prime}+1, \zeta\right), \ldots, \bar{C}\left(m^{\prime}+N_{\zeta}(1)-1, \zeta\right)$ by $\bar{C}\left(m^{\prime}-1, \zeta+1\right), \bar{C}\left(m^{\prime}, \zeta+1\right), \ldots, \bar{C}\left(m^{\prime}+N_{\zeta}(1)-2, \zeta+1\right)$, $1 \leq \zeta \leq d$

We set $\left\{N_{i}(s)\right\}_{2 \leq s \leq m^{\prime}, i=1,2, \ldots, d+s-1}$ as

$$
\begin{aligned}
& N_{d+1}(2):=N_{d}(1) \text {, } \\
& N_{d}(2):=N_{d-1}(1)+\#\left\{1 \leq j \leq m-m^{\prime} \mid K_{d}^{(j)}-d=m^{\prime}-1\right\} \text {, } \\
& N_{d-1}(2):=N_{d-2}(1)+\#\left\{1 \leq j \leq m-m^{\prime} \mid K_{d-1}^{(j)}-(d-1)=m^{\prime}-1\right\} \text {, } \\
& N_{2}(2):=N_{1}(1)+\#\left\{1 \leq j \leq m-m^{\prime} \mid K_{2}^{(j)}-2=m^{\prime}-1\right\} \text {, } \\
& N_{1}(2):=\#\left\{1 \leq j \leq m-m^{\prime} \mid K_{1}^{(j)}-1=m^{\prime}-1\right\} \text {, } \\
& N_{d+2}(3):=N_{d+1}(2) \text {, } \\
& N_{d+1}(3):=N_{d}(2) \text {, } \\
& N_{d}(3):=N_{d-1}(2)+\#\left\{1 \leq j \leq m-m^{\prime} \mid K_{d}^{(j)}-d=m^{\prime}-2\right\} \text {, } \\
& N_{d-1}(3):=N_{d-2}(2)+\#\left\{1 \leq j \leq m-m^{\prime} \mid K_{d-1}^{(j)}-(d-1)=m^{\prime}-2\right\} \text {, } \\
& N_{2}(3):=N_{1}(2)+\#\left\{1 \leq j \leq m-m^{\prime} \mid K_{2}^{(j)}-2=m^{\prime}-2\right\} \text {, } \\
& N_{1}(3):=\#\left\{1 \leq j \leq m-m^{\prime} \mid K_{1}^{(j)}-1=m^{\prime}-2\right\} \text {, } \\
& N_{m^{\prime}+d-1}\left(m^{\prime}\right):=N_{m^{\prime}+d-2}\left(m^{\prime}-1\right) \text {, } \\
& N_{m^{\prime}+d-2}\left(m^{\prime}\right):=N_{m^{\prime}+d-3}\left(m^{\prime}-1\right) \text {, } \\
& N_{d+1}\left(m^{\prime}\right):=N_{d}\left(m^{\prime}-1\right), \\
& N_{d}\left(m^{\prime}\right):=N_{d-1}\left(m^{\prime}-1\right)+\#\left\{1 \leq j \leq m-m^{\prime} \mid K_{d}^{(j)}-d=1\right\} \text {, }
\end{aligned}
$$




$$
\begin{aligned}
& N_{2}\left(m^{\prime}\right):=N_{1}\left(m^{\prime}-1\right)+\#\left\{1 \leq j \leq m-m^{\prime} \mid K_{2}^{(j)}-2=1\right\}, \\
& N_{1}\left(m^{\prime}\right):=\#\left\{1 \leq j \leq m-m^{\prime} \mid K_{1}^{(j)}-1=1\right\} .
\end{aligned}
$$

For example, if $K_{d}^{\left(m-m^{\prime}\right)}-d=m^{\prime}$, then the factor $\bar{C}\left(m^{\prime}, d\right)$ in $Y$ is acted by $\tilde{e}_{m^{\prime}+d-1} \cdots \tilde{e}_{d+2} \tilde{e}_{d+1} \tilde{e}_{d}$ since $N_{m^{\prime}+d-1}\left(m^{\prime}\right)=N_{m^{\prime}+d-2}\left(m^{\prime}-1\right)=N_{m^{\prime}+d-3}\left(m^{\prime}-2\right)=\cdots=N_{d}(1)$, and we obtain

$$
\tilde{e}_{m^{\prime}+d-1} \cdots \tilde{e}_{d+2} \tilde{e}_{d+1} \tilde{e}_{d} \cdot \bar{C}\left(m^{\prime}, d\right)=\bar{C}\left(0, m^{\prime}+d\right)=\bar{C}\left(R_{d}^{\left(m-m^{\prime}\right)}, K_{d}^{\left(m-m^{\prime}\right)}\right),
$$

by (6.24) and (6.26). In general, if $K_{i}^{(j)}-i=m^{\prime}-\zeta, 0 \leq \zeta \leq m^{\prime}-1$, then the factor $\bar{C}(m-j, i)$ in $Y$ is acted by $\tilde{e}_{m^{\prime}-\zeta+i-1} \cdots \tilde{e}_{i+2} \tilde{e}_{i+1} \tilde{e}_{i}$, and we obtain

$$
\tilde{e}_{m^{\prime}-\zeta+i-1} \cdots \tilde{e}_{i+2} \tilde{e}_{i+1} \tilde{e}_{i} \bar{C}(m-j, i)=\bar{C}\left(m-m^{\prime}-j+\zeta, m^{\prime}-\zeta+i\right)=\bar{C}\left(R_{i}^{(j)}, K_{i}^{(j)}\right),
$$

which means (6.36). Therefore, we get (6.33).

Example 6.18. We set $r=9, m=6, m^{\prime}=3$ and $d=4$. Let us see that we can obtain

$$
\begin{array}{r}
\bar{C}(6,1) \cdot \bar{C}(6,2) \cdot \bar{C}(5,4) \cdot \bar{C}(4,6) \\
M:=\bar{C}(4,2) \cdot \bar{C}(4,3) \cdot \bar{C}(4,4) \cdot \bar{C}(3,6) \\
\bar{C}(1,4) \cdot \bar{C}(1,5) \cdot \bar{C}(1,6) \cdot \bar{C}(1,7)
\end{array}
$$

from

$$
\begin{array}{r}
\bar{C}(6,1) \cdot \bar{C}(6,2) \cdot \bar{C}(6,3) \cdot \bar{C}(6,4) \\
Y:=\bar{C}(5,1) \cdot \bar{C}(5,2) \cdot \bar{C}(5,3) \cdot \bar{C}(5,4) \\
\bar{C}(4,1) \cdot \bar{C}(4,2) \cdot \bar{C}(4,3) \cdot \bar{C}(4,4) .
\end{array}
$$

by applying $\left\{\tilde{e}_{i}^{N_{i}(s)}\right\}$ in the proof of Theorem 5.6, that is

$$
\begin{aligned}
& \underbrace{\tilde{e}_{1}^{\left(N_{1}(3)\right)} \tilde{e}_{2}^{\left(N_{2}(3)\right)} \tilde{e}_{3}^{\left(N_{3}(3)\right)} \tilde{e}_{4}^{\left(N_{4}(3)\right)} \tilde{e}_{5}^{\left(N_{5}(3)\right)} \tilde{e}_{6}^{\left(N_{6}(3)\right)}}_{3^{\text {rd }} \text { cycle }} \cdot \underbrace{\tilde{e}_{1}^{\left(N_{1}(2)\right)} \tilde{e}_{2}^{\left(N_{2}(2)\right)} \tilde{e}_{3}^{\left(N_{3}(2)\right)} \tilde{e}_{4}^{\left(N_{4}(2)\right)} \tilde{e}_{5}^{\left(N_{5}(2)\right)}}_{2^{\text {nd }} \text { cycle }} \\
& \cdot \underbrace{\tilde{e}_{1}^{\left(N_{1}(1)\right)} \tilde{e}_{2}^{\left(N_{2}(1)\right)} \tilde{e}_{3}^{\left(N_{3}(1)\right)} \tilde{e}_{4}^{\left(N_{4}(1)\right)}}_{1^{\text {st }} \text { cycle }} \cdot Y=M .
\end{aligned}
$$

We set $M=\prod_{j=1}^{3} \prod_{i=1}^{4} \bar{C}\left(R_{i}^{(j)}, K_{i}^{(j)}\right)$, that is, $K_{1}^{(1)}=1, K_{2}^{(1)}=2, K_{3}^{(1)}=4, K_{4}^{(1)}=6, K_{1}^{(2)}=2$, $K_{2}^{(2)}=3, \ldots, K_{4}^{(3)}=7$.

To change the factor $\bar{C}(4,4)$ of $Y$ into $\bar{C}(1,7)$ of $M$, we need to apply $\tilde{e}_{6} \tilde{e}_{5} \tilde{e}_{4}$ to $\bar{C}(4,4)$. In contrast, to change the factors $\bar{C}(5,4)$ and $\bar{C}(6,4)$ of $Y$ into $\bar{C}(3,6)$ and $\bar{C}(4,6)$, we need to apply $\tilde{e}_{5} \tilde{e}_{4}$ to $\bar{C}(5,4)$ and $\bar{C}(6,4)$. Thus, $\bar{C}(4,4)$ must be changed to $\bar{C}(3,5)$ by the action of $\tilde{e}_{4}^{\left(N_{4}(1)\right)}$ in first cycle, and $\bar{C}(5,4), \bar{C}(6,4)$ do not have to be changed at the first cycle. So we set $N_{4}(1):=1=\#\left\{j \mid K_{4}^{(j)}-4=3\right\}$. Similarly, we set $N_{3}(1)=N_{2}(1)=N_{1}(1)=1$, and the factors $\bar{C}(4,1), \bar{C}(4,2), \bar{C}(4,3)$ of $Y$ is changed to $\bar{C}(3,2), \bar{C}(3,3), \bar{C}(3,4)$ by the action of first cycle.

Next, to obtain $M$, the factor $\bar{C}(3,5)$ in $\tilde{e}_{1}^{\left(N_{1}(1)\right)} \tilde{e}_{2}^{\left(N_{2}(1)\right)} \tilde{e}_{3}^{\left(N_{3}(1)\right)} \tilde{e}_{4}^{\left(N_{4}(1)\right)} \cdot Y$ must be changed to $\bar{C}(2,6)$ at the second cycle. Thus, we set $N_{5}(2):=1=N_{4}(1)$. The factor $\bar{C}(3,4)$ must be changed to $\bar{C}(2,5)$ by the action of $\tilde{e}_{4}^{\left(N_{4}(2)\right)}$ in second cycle, and the factors $\bar{C}(5,4)$ and $\bar{C}(6,4)$ must also be changed at the second cycle. Hence, we set $N_{4}(2):=3=N_{3}(1)+\#\left\{j \mid K_{4}^{(j)}-4=2\right\}$. Similarly, we set $N_{3}(2)=1, N_{2}(2)=1, N_{1}(2)=0, N_{6}(3)=1, N_{5}(3)=3, N_{4}(3)=1, N_{3}(3)=3$, $N_{2}(3)=1$ and $N_{1}(3)=1$. Then we get (6.37). 
Example 6.19. We use the same setting in Example 6.15: $u=s_{1} s_{2} s_{3} s_{4} s_{1} s_{2} s_{3} s_{1} s_{2} s_{1}, k=6$, $m=3$ and $m^{\prime}=2$. We have $u_{\leq 6}=s_{1} s_{2} s_{3} s_{4} s_{1} s_{2}$. Let

$$
Y:=\frac{1}{\tau_{l_{2}+2}} \in \mathcal{Y}
$$

which has weight $-\Lambda_{2}$. By Theorem 5.6, we obtain

$$
\begin{aligned}
\Delta^{L}(6 ; \mathbf{i})(\tau) & =\sum_{x \in B_{u_{\leq 6}}^{-}\left(-\Lambda_{2}\right)} \mu_{Y}(x)=Y+\tilde{e}_{2} Y+\tilde{e}_{1} \tilde{e}_{2} Y+\tilde{e}_{3} \tilde{e}_{2} Y+\tilde{e}_{3} \tilde{e}_{1} \tilde{e}_{2} Y+\tilde{e}_{2} \tilde{e}_{3} \tilde{e}_{1} \tilde{e}_{2} Y \\
& =\frac{1}{\tau_{l_{2}+2}}+\frac{\tau_{l_{1}+2}}{\tau_{l_{2}+1} \tau_{l_{1}+3}}+\frac{\tau_{l_{1}+1}}{\tau_{l_{1}+3}}+\frac{\tau_{l_{0}+3}}{\tau_{l_{2}+1} \tau_{l_{0}+4}}+\frac{\tau_{l_{1}+1} \tau_{l_{0}+3}}{\tau_{l_{1}+2} \tau_{l_{0}+4}}+\frac{\tau_{l_{0}+2}}{\tau_{l_{0}+4}} .
\end{aligned}
$$

\subsection{The proof of Theorem 5.7}

Let us prove Theorem 5.7. Suppose that $i_{k}=d=1$.

Proof of Theorem 5.7. By Proposition 6.8, we have

$$
\Delta^{L}(k ; \mathbf{i})(\tau)=\sum_{p \in X_{1}\left(m, m^{\prime}\right)} Q(p)
$$

The set $X_{1}\left(m, m^{\prime}\right)$ consists of paths $p$ as in the form

$$
p=(m, 1) \rightarrow\left(m-1, a^{(1)}\right) \rightarrow\left(m-2, a^{(2)}\right) \rightarrow \cdots \rightarrow\left(1, a^{(m-1)}\right) \rightarrow\left(0, m^{\prime}+1\right)
$$

such that $a^{(s+1)}=a^{(s)}$ or $a^{(s)}+1,0 \leq s \leq m-1$. Here, $a^{(0)}:=1, a^{(m)}:=m^{\prime}+1$.

By Lemma 6.10, we obtain

$$
\#\left\{s \mid a^{(s+1)}=a^{(s)}+1,0 \leq s \leq m-1\right\}=m^{\prime} .
$$

Set $\left\{s \mid a^{(s+1)}=a^{(s)}+1,0 \leq s \leq m-1\right\}:=\left\{j_{1}, \ldots, j_{m^{\prime}}\right\}, 0 \leq j_{1}<\cdots<j_{m^{\prime}} \leq m-1$. Then we have

$$
\begin{aligned}
& a^{(0)}=1, \quad a^{(1)}=1, \quad a^{(2)}=1, \quad \ldots, \quad a^{\left(j_{1}\right)}=1, \quad a^{\left(j_{1}+1\right)}=2, \\
& a^{\left(j_{1}+2\right)}=2, \quad a^{\left(j_{1}+3\right)}=2, \quad \ldots, \quad a^{\left(j_{2}\right)}=2, \quad a^{\left(j_{2}+1\right)}=3, \\
& a^{\left(j_{2}+2\right)}=3, \quad a^{\left(j_{2}+3\right)}=3, \quad \ldots, \quad a^{\left(j_{3}\right)}=3, \quad a^{\left(j_{3}+1\right)}=4,
\end{aligned}
$$

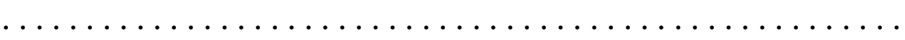

$$
\begin{aligned}
& a^{\left(j_{\nu}+2\right)}=\nu+1, \quad a^{\left(j_{\nu}+3\right)}=\nu+1, \quad \ldots, \quad a^{\left(j_{\nu+1}\right)}=\nu+1, \quad a^{\left(j_{\nu+1}+1\right)}=\nu+2, \\
& a^{\left(j_{m^{\prime}}+2\right)}=m^{\prime}+1, \quad a^{\left(j_{m^{\prime}}+3\right)}=m^{\prime}+1, \quad \ldots, \quad a^{(m)}=m^{\prime}+1 .
\end{aligned}
$$

Therefore, Definition 6.4(ii) means

$$
Q(p)=\prod_{i=0}^{j_{1}-1} \frac{1}{\tau_{l_{m-1-i}+1}} \prod_{i=j_{1}+1}^{j_{2}-1} \frac{\tau_{l_{m-1-i}+1}}{\tau_{l_{m-1-i}+2}} \cdots \prod_{i=j_{m^{\prime}}+1}^{m-1} \frac{\tau_{l_{m-1-i}+m^{\prime}}}{\tau_{l_{m-1-i}+m^{\prime}+1}} .
$$

Conversely, for a given $\left\{j_{1}, \ldots, j_{m^{\prime}}\right\}, 0 \leq j_{1}<\cdots<j_{m^{\prime}} \leq m-1$, we can constitute a path $p$ as in (6.39). Hence, by (6.38), we proved our claim. 


\section{Acknowledgements}

The authors would like to acknowledge the referees for giving them relevant advice and suggestion to improve this article. T.N. is supported in part by JSPS Grants in Aid for Scientific Research $\sharp 22540031, \sharp 15 K 04794$.

\section{References}

[1] Berenstein A., Fomin S., Zelevinsky A., Cluster algebras. III. Upper bounds and double Bruhat cells, Duke Math. J. 126 (2005), 1-52, math.RT/0305434.

[2] Berenstein A., Zelevinsky A., Tensor product multiplicities, canonical bases and totally positive varieties, Invent. Math. 143 (2001), 77-128, math.RT/9912012.

[3] Fomin S., Zelevinsky A., Double Bruhat cells and total positivity, J. Amer. Math. Soc. 12 (1999), 335-380, math.RT/9802056.

[4] Gekhtman M., Shapiro M., Vainshtein A., Cluster algebras and Poisson geometry, Mathematical Surveys and Monographs, Vol. 167, Amer. Math. Soc., Providence, RI, 2010.

[5] Hong J., Kang S.-J., Introduction to quantum groups and crystal bases, Graduate Studies in Mathematics, Vol. 42, Amer. Math. Soc., Providence, RI, 2002.

[6] Kashiwara M., On crystal bases of the $Q$-analogue of universal enveloping algebras, Duke Math. J. 63 (1991), $465-516$.

[7] Kashiwara M., Bases cristallines des groupes quantiques, Cours Spécialisés, Vol. 9, Société Mathématique de France, Paris, 2002.

[8] Kashiwara M., Realizations of crystals, in Combinatorial and Geometric Representation Theory (Seoul, 2001), Contemp. Math., Vol. 325, Amer. Math. Soc., Providence, RI, 2003, 133-139, math.QA/0202268.

[9] Kashiwara M., Nakashima T., Crystal graphs for representations of the $q$-analogue of classical Lie algebras, J. Algebra 165 (1994), 295-345.

[10] Nakajima H., $t$-analogs of $q$-characters of quantum affine algebras of type $A_{n}, D_{n}$, in Combinatorial and Geometric Representation Theory (Seoul, 2001), Contemp. Math., Vol. 325, Amer. Math. Soc., Providence, RI, 2003, 141-160, math.QA/0204184.

[11] Nakashima T., Decorations on geometric crystals and monomial realizations of crystal bases for classical groups, J. Algebra 399 (2014), 712-769, arXiv:1301.7301. 